\title{
Two-scale div-curl lemma
}

\author{
Augusto Visintin
}

\begin{abstract}
The div-curl lemma, one of the basic results of the theory of compensated compactness of Murat and Tartar, does not take over to the case in which the two factors two-scale converge in the sense of Nguetseng. A suitable modification of the differential operators however allows for this extension. The argument follows the lines of a well-known paper of F. Murat of 1978, and uses a two-scale extension of the Fourier transform. This result is also extended to time-dependent functions, and is applied to a two-scale formulation of the Maxwell system of electromagnetism, that accounts for the energy embedded in both coarse- and fine-scale oscillations.
\end{abstract}

Mathematics Subject Classification (2000): 35B27 (primary); 35J20, 74Q (secondary).

\section{Introduction}

This paper deals with the extention of the classical div-curl lemma to the framework of two-scale convergence. Let us first review both notions.

\section{Two-scale convergence}

Let us fix any $N \geq 1$ and set $Y:=\left[0,1\left[{ }^{N}\right.\right.$. In [22] Nguetseng introduced the following concept, that was then studied in detail by Allaire [1] and others. A bounded sequence $\left\{u_{\varepsilon}\right\}$ of $L^{2}\left(\mathbb{R}^{N}\right)$ is said (weakly) two-scale convergent to $u \in$ $L^{2}\left(\mathbb{R}^{N} \times Y\right)$ whenever

$$
\lim _{\varepsilon \rightarrow 0} \int_{\mathbb{R}^{N}} u_{\varepsilon}(x) \psi(x, x / \varepsilon) d x=\iint_{\mathbb{R}^{N} \times Y} u(x, y) \psi(x, y) d x d y,
$$

for any smooth function $\psi: \mathbb{R}^{N} \times \mathbb{R}^{N} \rightarrow \mathbb{R}$ that is $Y$-periodic with respect to the second argument. We then write $u_{\varepsilon} \underset{2}{\longrightarrow} u$ in $L^{2}\left(\mathbb{R}^{N} \times Y\right)$. This definition is

This research was partially supported by the projects "Mathematical modelling and analysis of free boundary problems" and "Free boundary problems, phase transitions and models of hysteresis" of Italian M.I.U.R.

Received April 26, 2006; accepted in revised form May 4, 2007. 
generalized to functions defined on a domain $\Omega$ of $\mathbb{R}^{N}$ just by extending them with vanishing value outside that domain; the extension to either vector- or complex- valued functions is also straightforward. This notion may account for the occurrence of a fine-scale periodic structure, and has been applied to a number of homogenization problems: $[1,3,10,22,23]$ and many others. For periodic homogenization this method is indeed an alternative to the classical energy method of Tartar, see e.g. $[2,6,11,15,21,24,26-29]$.

\section{Div-curl lemma}

We shall deal throughout with complex-valued functions; for instance by $L^{2}(\Omega)$ we shall denote the Hilbert space of square integrable functions $\Omega \rightarrow \mathbb{C}$. For any $u, v \in \mathbb{C}^{N}$ let us set

$$
u \cdot v:=\sum_{j=1}^{N} u_{j} v_{j}, \quad u \times v:=\left\{u_{j} v_{k}-u_{k} v_{j}: j, k=1, \ldots, N\right\}
$$

(thus $|u|^{2}=u \cdot u^{*}$ ). Denoting the partial derivative with respect to $x_{j}$ by $\nabla_{j}$ for $j=1, \ldots, N$, we also define the divergence, $\nabla \cdot$, and the curl, $\nabla \times$ :

$\nabla \cdot v:=\sum_{j=1}^{N} \nabla_{j} v_{j}, \quad \nabla \times v:=\left\{\nabla_{j} v_{k}-\nabla_{k} v_{j}: j, k=1, \ldots, N\right\} \quad \forall v \in \mathcal{D}^{\prime}\left(\mathbb{R}^{N}\right)$.

In the paper [18] that appeared in this journal in 1978, Murat established the first results of compensated compactness due to his collaboration with Tartar, see also [19-21, 26-29]. As a starting point he proved the next theorem.

Theorem 1.1 (div-curl lemma). Let $\Omega$ be a (possibly unbounded) domain of $\mathbb{R}^{N}$, $\left\{u_{\varepsilon}\right\}$ and $\left\{w_{\varepsilon}\right\}$ be two sequences of $L^{2}(\Omega)^{N}$ such that $\nabla \times u_{\varepsilon} \in L^{2}(\Omega)^{N^{2}}$ and $\nabla \cdot w_{\varepsilon} \in L^{2}(\Omega)$ for any $\varepsilon$. If

$$
\begin{gathered}
\left\{\nabla \times u_{\varepsilon}\right\} \text { is bounded in } L^{2}(\Omega)^{N^{2}}, \quad\left\{\nabla \cdot w_{\varepsilon}\right\} \text { is bounded in } L^{2}(\Omega), \\
u_{\varepsilon} \rightarrow \bar{u}, \quad w_{\varepsilon} \rightarrow \bar{w} \quad \text { weakly in } L^{2}(\Omega)^{N}
\end{gathered}
$$

then $u_{\varepsilon} \cdot w_{\varepsilon} \rightarrow \bar{u} \cdot \bar{w}$ weakly star in $C_{c}^{0}(\Omega)^{\prime}$, i.e.,

$$
\int_{\Omega} u_{\varepsilon}(x) \cdot w_{\varepsilon}(x) \varphi(x) d x \rightarrow \int_{\Omega} \bar{u}(x) \cdot \bar{w}(x) \varphi(x) d x \quad \forall \varphi \in C_{c}^{0}(\Omega) .
$$

However $u_{\varepsilon} \cdot w_{\varepsilon}$ need not weakly converge in $L^{1}(\Omega)$, see [19] for a counterexample. On the other hand, if in (1.2) and (1.3) $L^{2}$ is replaced by $L_{\text {loc }}^{2}$ the extension is trivial. This theorem was generalized in several ways, $c f$. [18-21, 26-29]; in particular in [19] the condition (1.2) was replaced by the weaker assumption

$$
\begin{aligned}
& \left\{\nabla \times u_{\varepsilon}\right\}\left(\left\{\nabla \cdot w_{\varepsilon}\right\}, \text { respectively }\right) \text { is in a compact subset of } \\
& H^{-1}(\Omega)^{N^{2}}\left(H^{-1}(\Omega), \text { respectively }\right)
\end{aligned}
$$


Results like this and more general theorems of compensated compactness proved to be useful for the analysis and the homogenization of a large number of partial differential equations. Concerning time-dependent functions, classical compactness theorems were also established by Aubin and others, $c f$. e.g. [4, 25] and [17, Section 1.5].

\section{Towards a two-scale extension}

Let us now replace the hypothesis (1.3) by the condition

$$
u_{\varepsilon} \underset{2}{\rightarrow} u, \quad w_{\varepsilon} \underset{2}{\rightarrow} w \quad \text { in } L^{2}(\Omega \times Y)^{N},
$$

which yields (1.3) for the limit functions $\bar{u}:=\int_{Y} u(\cdot, y) d y$ and $\bar{w}:=\int_{Y} w(\cdot, y) d y$. It seems natural to guess and test the validity of the following two-scale extensions of (1.4):

$$
\begin{aligned}
& \int_{\Omega} u_{\varepsilon}(x) \cdot w_{\varepsilon}(x) \varphi(x) d x \rightarrow \iint_{\Omega \times Y} u(x, y) \cdot w(x, y) \varphi(x) d x \quad \forall \varphi \in C_{c}^{0}(\Omega), \\
& \int_{\Omega} u_{\varepsilon}(x) \cdot w_{\varepsilon}(x) \psi(x, x / \varepsilon) d x \rightarrow \iint_{\Omega \times Y} u(x, y) \cdot w(x, y) \psi(x, y) d x d y
\end{aligned}
$$

for any $\psi \in C_{c}^{0}\left(\Omega ; C^{0}\left(\mathbb{R}^{N}\right)\right)$ that is $Y$-periodic with respect to

the second argument.

(1.7) easily follows from (1.2), (1.4) and (1.6). Indeed (1.2) and (1.6) yield $\nabla_{y} \times u=$ 0 and $\nabla_{y} \cdot w=0$ in the sense of distributions, whence ( $c f$. [34])

$$
\iint_{\Omega \times Y} u(x, y) \cdot w(x, y) \varphi(x) d x=\int_{\Omega} \bar{u}(x) \cdot \bar{w}(x) \varphi(x) d x \quad \forall \varphi \in C_{c}^{0}(\Omega) ;
$$

thus (1.4) yields (1.7). On the other hand in [9] it was shown that (1.2) and (1.6) do not entail (1.8). This is due to the lack of control on the $x$-derivatives of the two-scale limit functions $u$ and $w$, which allows for the onset of oscillations of frequency slower than $1 / \varepsilon$; see also [34].

\section{Two-scale-approximation of partial differential operators}

In this paper we overcome the difficulty that we just outlined by amending the hypothesis (1.2). In view of illustrating this result, let us first define the shift operator $\left(\tau_{h} v\right)(x):=v(x+h)$ for any $x, h \in \mathbb{R}^{N}$, denote by $e_{j}$ the unit vector of the $j$-th axis, and set

$$
\nabla_{\varepsilon j}:=\left(\tau_{\varepsilon e_{j}}-I\right) / \varepsilon \quad \text { for } j=1, \ldots, N, \quad \nabla_{\varepsilon}:=\left(\nabla_{\varepsilon 1}, \ldots, \nabla_{\varepsilon N}\right) .
$$

(Obviously $\nabla_{\varepsilon} v=0$ in the whole $\mathbb{R}^{N}$ if and only if $v$ is $Y$-periodic.) The operators $\nabla_{\varepsilon}$ and $\varepsilon \nabla$ approximate the partial gradients $\nabla_{x}$ and $\nabla_{y}$ in the sense of the next statement. 
Proposition 1.2 ( [31]). Let $\left\{v_{\varepsilon}\right\}$ be a sequence of $L_{\mathrm{loc}}^{2}(\Omega)$. If

$$
\begin{gathered}
v_{\varepsilon} \underset{2}{ } v \quad \text { in } L^{2}(\Omega \times Y), \\
\nabla_{\varepsilon} v_{\varepsilon} \underset{2}{\rightarrow} z_{1}, \quad \varepsilon \nabla v_{\varepsilon} \underset{2}{\rightarrow} z_{2} \quad \text { in } L^{2}(\Omega \times Y)^{N},
\end{gathered}
$$

then

$$
v \in H^{1}(\Omega \times Y), \quad z_{1}=\nabla_{x} v, \quad z_{2}=\nabla_{y} v \quad \text { a.e. in } \Omega \times Y .
$$

An analogous result applies to vector-valued functions, if $\nabla_{\varepsilon}$ is replaced either by $\nabla_{\varepsilon} \times$ or by $\nabla_{\varepsilon} \cdot$, and similarly if $\varepsilon \nabla$ is replaced either by $\varepsilon \nabla \times$ or by $\varepsilon \nabla \cdot$. As

$$
\begin{aligned}
\nabla_{\varepsilon j} v(x)=\int_{0}^{1} \nabla_{x_{j}} v\left(x+\varepsilon \lambda e_{j}\right) d \lambda & =\nabla_{x_{j}} \int_{0}^{1} v\left(x+\varepsilon \lambda e_{j}\right) d \lambda \\
& =\nabla_{x_{j}}\left(\frac{1}{\varepsilon} \int_{0}^{\varepsilon} v\left(x+\lambda e_{j}\right) d \lambda\right),
\end{aligned}
$$

the operators $\nabla_{\varepsilon}$ or by $\varepsilon \nabla$ may be regarded as coarse- and fine-scale approximate derivatives, respectively.

\section{Two-scale div-curl lemma}

Let us set

$$
G_{\varepsilon}:=\left(\nabla_{\varepsilon}, \varepsilon \nabla\right), \quad G:=\left(\nabla_{x}, \nabla_{y}\right)
$$

$$
\begin{aligned}
& \forall \varepsilon>0, \forall v=\left(v_{1}, v_{2}\right): \mathbb{R}^{N} \rightarrow \mathbb{R}^{N} \times \mathbb{R}^{N}, \quad G_{\varepsilon} v:=\left(\begin{array}{cc}
\nabla_{\varepsilon} v_{1} & \varepsilon \nabla v_{1} \\
\nabla_{\varepsilon} v_{2} & \varepsilon \nabla v_{2}
\end{array}\right), \\
& G_{\varepsilon} \times v:=\text { antisymmetric part of } G_{\varepsilon} v, \quad G_{\varepsilon} \cdot v:=\text { trace of } G_{\varepsilon} v
\end{aligned}
$$

$\forall Y$-periodic $z=\left(z_{1}, z_{2}\right): \mathbb{R}^{N} \times Y \rightarrow \mathbb{R}^{N} \times \mathbb{R}^{N}, \quad G z:=\left(\begin{array}{cc}\nabla_{x} z_{1} & \nabla_{y} z_{1} \\ \nabla_{x} z_{2} & \nabla_{y} z_{2}\end{array}\right)$,

$G \times z:=$ antisymmetric part of $G z, \quad G \cdot z:=$ trace of $G z$.

In Theorem 5.1 we shall prove (1.8) under the assumption that (1.6) is fulfilled and that

$$
\left\{G_{\varepsilon} \times u_{\varepsilon}\right\} \text { is bounded in } L_{\mathrm{loc}}^{2}(\Omega)^{4 N^{2}}, \quad\left\{G_{\varepsilon} \cdot w_{\varepsilon}\right\} \text { is bounded in } L_{\mathrm{loc}}^{2}(\Omega) .
$$

Notice that (1.16) entails $G \times u \in L_{\text {loc }}^{2}(\Omega \times Y)^{4 N^{2}}$ and $G \cdot w \in L_{\text {loc }}^{2}(\Omega \times Y)$, for after Proposition 1.2 the operators $G_{\varepsilon} \times$ and $G_{\varepsilon} \cdot$ respectively approximate $G \times$ and $G$. 
in the sense of weak two-scale convergence. However it is clear that (1.16) does not entail

$$
\left\{\nabla \times u_{\varepsilon}\right\} \text { is bounded in } L_{\text {loc }}^{2}(\Omega)^{N^{2}}, \quad\left\{\nabla \cdot w_{\varepsilon}\right\} \text { is bounded in } L_{\text {loc }}^{2}(\Omega) .
$$

Moreover (less obviously) the converse implication also fails: (1.17) $\not \Rightarrow$ (1.16), for otherwise Theorem 5.1 would not be consistent with the negative result of [9]. Actually, by the mean value theorem the $L_{\text {loc }}^{2}$-boundedness of $\nabla u_{\varepsilon}$ entails that of $\nabla_{\varepsilon} u_{\varepsilon}$. But the $L_{\text {loc }}^{2}$-boundedness of $\nabla \times u_{\varepsilon}$ and $\nabla \cdot w_{\varepsilon}$ does not entail that of $\nabla_{\varepsilon} \times u_{\varepsilon}$ and $\nabla_{\varepsilon} \cdot w_{\varepsilon}$, because the mean value theorem fails for the curl and divergence operators.

Alike the classical div-curl lemma, the argument of Theorem 5.1 is also based on the Fourier transform. This transform is here applied to the approximating functions of a single (vector) variable and to the limit functions of two (vector) variables; the definition of an approximate two-scale Fourier transform seems then convenient.

\section{Two-scale Maxwell system}

The two-scale div-curl lemma may be applied e.g. to a two-scale formulation of the Maxwell equations of electromagnetism, in which the fields depend on both variables $x, y$, and the single-scale operator $\nabla$ is replaced by the two-scale differential operator $G$ for $N=3$; thus $G=\left(\nabla_{x}, \nabla_{y}\right)=\left(\nabla_{1}, \ldots, \nabla_{6}\right)$. This setting is quite different from that issued from the application of two-scale convergence to the standard Maxwell system, $c f$. e.g. [33], and applies if the fields have $O(1)$ variations at the $\varepsilon$ length-scale, despite of the occurrence of vector space derivatives.

By standard notation, let us denote the relevant electromagnetic fields by $B, H$, $D, E, J$ and so on, and identify the curl of vector fields of $\mathbb{R}^{3}$ with elements of $\mathbb{R}^{3}$, rather than $3 \times 3$ tensors. Assuming the Ohm law $E=\rho J$ (the resistivity $\rho$ being a positive constant, say), the single-scale Maxwell equations without displacement current $\partial D / \partial t$ are easily reduced to the single equation

$$
\left.\frac{\partial B_{i}}{\partial t}+\rho \sum_{j=1}^{3} \nabla_{j}\left(\nabla_{i} H_{j}-\nabla_{j} H_{i}\right)=f_{i} \quad \text { in } \mathbb{R}^{3} \times\right] 0, T[(i=1,2,3),
$$

for a prescribed source field $f$. We propose to replace the 3-component vector fields $B$ and $H$ by the 6-component fields

$$
B:=\left(B_{a}, B_{b}\right)=\left(B_{1}, \ldots, B_{6}\right), \quad H:=\left(H_{a}, H_{b}\right)=\left(H_{1}, \ldots, H_{6}\right)
$$

(the indices $a$ and $b$ respectively refer to the triplets $(1,2,3)$ and $(4,5,6))$, and then replace (1.18) by the two-scale equation

$$
\left.\frac{\partial B_{i}}{\partial t}+\rho \sum_{j=1}^{6} \nabla_{j}\left(\nabla_{i} H_{j}-\nabla_{j} H_{i}\right)=f_{i} \quad \text { in } \mathbb{R}^{3} \times Y \times\right] 0, T[(i=1, \ldots, 6),
$$


that is, setting $f=\left(f_{a}, f_{b}\right)=\left(f_{1}, \ldots, f_{6}\right)$,

$$
\left\{\begin{array}{cc}
\frac{\partial B_{a}}{\partial t}+\rho \nabla_{x} & \times \nabla_{x} \times H_{a} \\
& +\rho \nabla_{x}\left(\nabla_{y} \cdot H_{b}\right)-\rho \Delta_{y} H_{a}=f_{a}
\end{array} \quad \text { in } \mathbb{R}^{3} \times Y \times\right] 0, T[.
$$

This entails the two-scale Gauss law

$$
\left.\nabla_{x} \cdot B_{a}+\nabla_{y} \cdot B_{b}=0 \quad \text { in } \mathbb{R}^{3} \times Y \times\right] 0, T[,
$$

provided that this holds at the initial instant. Under natural side-conditions we also derive a two-scale extension of the energy integral, $c f .(7.20)$; this accounts for the energy embedded in both coarse- and fine-scale oscillations. The regularity properties

$$
\nabla_{x} \times H_{a}, \nabla_{y} \times H_{b} \in L^{2}\left(\mathbb{R}^{3} \times Y \times\right] 0, T[)^{3}
$$

are then naturally associated to the system (1.19).

We then propose to approximate the system (1.19) by replacing $\nabla_{x}$ and $\nabla_{y}$ respectively by $\nabla_{\varepsilon}$ and $\varepsilon \nabla$ for any $\varepsilon>0$, consistently with Proposition 1.2. In this setting the two-scale div-curl lemma finds a natural application, as the classical div-curl lemma does in the single-scale framework. For instance this is the case if a maximal monotone relation is assumed between $B$ and $H$.

Although here we confine ourselves to the Maxwell system, the two-scale formulation can be applied to several other partial differential equations. Actually the Maxwell example is somehow problematic due to the occurrence of the curl operator; in particular the doubling of the components is suggested by the necessity of deriving the Gauss law, $c f$. (1.21). For other P.D.E.'s the two-scale extension is simpler and will be studied apart.

\section{Plan of the paper}

In Section 2 we briefly review some fundamental properties of two-scale convergence. In Section 3 we introduce an $\varepsilon$-dependent variant, $\mathcal{F}_{\varepsilon}$, of the Fourier transform, $\mathcal{F}$, that seems appropriate for two-scale convergence. We then study the two-scale limit of $\mathcal{F}_{\varepsilon}$, and in Section 4 we apply it to differential operators, deriving properties that mimic those of $\mathcal{F}$. In Section 5 we prove the two-scale div-curl lemma along the lines of [18], using the transform $\mathcal{F}_{\varepsilon}$ instead of $\mathcal{F}$. In Section 6 we derive an analogous statement for time-dependent functions, extending Aubin-type compactness results. Finally in Section 7 we outline the two-scale formulation of the Maxwell system, and briefly illustrate the application of the two-scale div-curl lemma.

This work is part of a research that led the author to extend to two-scale convergence several properties of single-scale convergence [30-34]. Further work in 
this direction might include the extension of more general results of compensated compactness, and the application to the two-scale homogenization of P.D.E.'s, after approximation of the derivatives via the operators $\nabla_{\varepsilon}$ and $\varepsilon \nabla$.

\section{Two-scale convergence}

In this section we review some results of the theory of two-scale convergence, using an approach based on a scale decomposition, along the lines of [3, 7, 10, 16, 30,31].

We already set $Y:=\left[0,1\left[{ }^{N}\right.\right.$. We shall denote by $\mathcal{Y}$ the set $Y$ equipped with the topology of the $N$-dimensional unit torus, and identify any function on $\mathcal{Y}$ with its $Y$-periodic extension to $\mathbb{R}^{N}$. As far as integration is concerned, we may identify $\mathcal{Y}$ with $Y$; however this is not the case for continuity and differentiation. Thus $L^{p}(\mathcal{Y})=L^{p}(Y)$ but $C^{0}(\mathcal{Y}) \neq C^{0}(\bar{Y})$ and $W^{1, p}(\mathcal{Y}) \neq W^{1, p}(Y)$ for any $p \in$ $[1,+\infty]$.

For any $\varepsilon>0$ we decompose real numbers and real vectors as the sum of their integer and fractional parts:

$$
\begin{array}{lll}
\hat{n}(x):=\max \{n \in \mathbb{Z}: n \leq x\}, \quad \hat{r}(x):=x-\hat{n}(x)(\in[0,1[) & \forall x \in \mathbb{R}, \\
\mathcal{N}(x):=\left(\hat{n}\left(x_{1}\right), \ldots, \hat{n}\left(x_{N}\right)\right) \in \mathbb{Z}^{N}, \quad \mathcal{R}(x):=x-\mathcal{N}(x) \in Y & \forall x \in \mathbb{R}^{N} .
\end{array}
$$

Thus $x=\varepsilon[\mathcal{N}(x / \varepsilon)+\mathcal{R}(x / \varepsilon)]$ for any $x \in \mathbb{R}^{N} ; \varepsilon \mathcal{N}(x / \varepsilon)$ and $\mathcal{R}(x / \varepsilon)$ respectively represent coarse-scale and fine-scale variables with respect to the scale-ratio $\varepsilon$. Besides this two-scale decomposition (or periodic unfolding in the terminology of [10]), we define a two-scale composition:

$$
S_{\varepsilon}(x, y):=\varepsilon \mathcal{N}(x / \varepsilon)+\varepsilon y \quad \forall(x, y) \in \mathbb{R}^{N} \times Y, \forall \varepsilon>0 .
$$

Henceforth $\mathbb{Z}^{N}$ will be equipped with the counting measure, and any infinite sum over $\mathbb{Z}^{N}$ will be assumed to be absolutely convergent. We shall denote by $\mathcal{L}\left(\mathbb{R}^{N}\right)$ $\left(\mathcal{B}\left(\mathbb{R}^{N}\right)\right.$, respectively) the $\sigma$-algebra of Lebesgue- (Borel-, respectively) measurable subsets of $\mathbb{R}^{N}$, and define $\mathcal{L}(Y)$ and $\mathcal{B}(Y)$ similarly. We shall deal with complexvalued functions and with functions spaces over the field $\mathbb{C}$. The next lemma can easily be proved via a variable transformation.

Lemma 2.1 ( [31]). Let $f: \mathbb{R}^{N} \times \mathcal{Y} \rightarrow \mathbb{C}$ be such that

$f$ is measurable either with respect to $\mathcal{B}\left(\mathbb{R}^{N}\right) \otimes \mathcal{L}(\mathcal{Y})$

or with respect to $\mathcal{L}\left(\mathbb{R}^{N}\right) \otimes \mathcal{B}(\mathcal{Y})$, and

either $\|f\|_{L^{\infty}\left(\mathbb{R}^{N}\right)} \in L^{1}(\mathcal{Y})$ and $f$ has compact support,

$$
\text { or }\|f\|_{L^{\infty}(\mathcal{Y})} \in L^{1}\left(\mathbb{R}^{N}\right) \text {, }
$$

or, for some $m \in \mathbb{N}$,

$$
\begin{aligned}
& f(x, y)=\sum_{i=1}^{m} u_{i}(x) v_{i}(y) \quad \text { for almost all } x \in \mathbb{R}^{N} \text { and almost all } y \in \mathcal{Y}, \\
& \text { with } u_{i} \in L^{1}\left(\mathbb{R}^{N}\right), v_{i} \in L^{1}(\mathcal{Y}) \text { for } i=1, \ldots, m .
\end{aligned}
$$


For any $\varepsilon>0$, the functions $\mathbb{R}^{N} \rightarrow \mathbb{C}: x \mapsto f(x, x / \varepsilon)$ and $\mathbb{R}^{N} \times \mathcal{Y} \rightarrow \mathbb{C}:$ $(x, y) \mapsto f\left(S_{\varepsilon}(x, y), y\right)$ are then integrable, and

$$
\int_{\mathbb{R}^{N}} f(x, x / \varepsilon) d x=\iint_{\mathbb{R}^{N} \times \mathcal{Y}} f\left(S_{\varepsilon}(x, y), y\right) d x d y \quad \forall \varepsilon>0 .
$$

For any $p \in[1,+\infty]$ and any $\varepsilon>0$, the operator $g \mapsto g \circ S_{\varepsilon}$ is then a (nonsurjective) linear isometry $L^{p}\left(\mathbb{R}^{N}\right) \rightarrow L^{p}\left(\mathbb{R}^{N} \times \mathcal{Y}\right)$.

Throughout this paper by $\varepsilon$ we represent the generic element of an arbitrary but prescribed, vanishing sequence of positive numbers. For any sequence of measurable functions, $u_{\varepsilon}: \mathbb{R}^{N} \rightarrow \mathbb{C}$, and any measurable function, $u: \mathbb{R}^{N} \times \mathcal{Y} \rightarrow \mathbb{C}$, we say that $u_{\varepsilon}$ two-scale converges to $u$ (with respect to the prescribed sequence $\left.\left\{\varepsilon_{n}\right\}\right)$ in some specific sense, whenever $u_{\varepsilon} \circ S_{\varepsilon} \rightarrow u$ in the corresponding standard (i.e., single-scale) sense. For any $p \in[1,+\infty]$ we thus define strong and weak (weak star for $p=\infty$ ) two-scale convergence in $L^{p}\left(\mathbb{R}^{N} \times \mathcal{Y}\right.$ ), that we denote by $u_{\varepsilon} \underset{2}{\rightarrow} u, u_{\varepsilon} \overrightarrow{2} u, u_{\varepsilon} \frac{*}{2} u$ (respectively). We shall also use the symbols $\rightarrow, \rightarrow$, $*$ for strong, weak, weak star single-scale convergence. Thus

$$
\begin{aligned}
& u_{\varepsilon} \rightarrow \overrightarrow{2} \text { in } L^{p}\left(\mathbb{R}^{N} \times \mathcal{Y}\right) \Leftrightarrow u_{\varepsilon} \circ S_{\varepsilon} \rightarrow u \text { in } L^{p}\left(\mathbb{R}^{N} \times \mathcal{Y}\right), \\
& u_{\varepsilon} \underset{2}{\rightarrow} u \text { in } L^{p}\left(\mathbb{R}^{N} \times \mathcal{Y}\right) \Leftrightarrow u_{\varepsilon} \circ S_{\varepsilon} \rightarrow u \text { in } L^{p}\left(\mathbb{R}^{N} \times \mathcal{Y}\right), \\
& u_{\varepsilon} \underset{2}{*} u \text { in } L^{\infty}\left(\mathbb{R}^{N} \times \mathcal{Y}\right) \Leftrightarrow u_{\varepsilon} \circ S_{\varepsilon} * u \text { in } L^{\infty}\left(\mathbb{R}^{N} \times \mathcal{Y}\right) .
\end{aligned}
$$

For any domain $\Omega \subset \mathbb{R}^{N}$ two-scale convergence in $L^{p}(\Omega \times \mathcal{Y})$ is then easily defined by extending functions to $\mathbb{R}^{N} \backslash \Omega$ with vanishing value. The generalization of twoscale convergence to vector-valued functions is also straightforward. In [31] it is shown that (2.6) generalizes the original definitions of $[1,22]$. However it should be noticed that the weak two-scale convergence in $L^{1}\left(\mathbb{R}^{N} \times \mathcal{Y}\right)$ is not a very natural notion: it cannot be formulated as in (1.1), for the smooth functions are not dense in the dual space $L^{\infty}\left(\mathbb{R}^{N} \times \mathcal{Y}\right)$. The next statement relates two-scale and single-scale convergence.

Proposition 2.2 ([31]). Let $p \in\left[1,+\infty\left[\right.\right.$ and $\left\{u_{\varepsilon}\right\}$ be a sequence in $L^{p}\left(\mathbb{R}^{N}\right)$. Then:

$$
\begin{aligned}
& \text { whenever } u \text { is independent of } y \text {, } \\
& u_{\varepsilon} \rightarrow u \text { in } L^{p}\left(\mathbb{R}^{N}\right) \Leftrightarrow u_{\varepsilon} \rightarrow \underset{2}{ } u \text { in } L^{p}\left(\mathbb{R}^{N} \times \mathcal{Y}\right) \text {, } \\
& u_{\varepsilon} \underset{2}{\rightarrow} u \text { in } L^{p}\left(\mathbb{R}^{N} \times \mathcal{Y}\right) \Rightarrow u_{\varepsilon} \overrightarrow{2} u \text { in } L^{p}\left(\mathbb{R}^{N} \times \mathcal{Y}\right), \\
& u_{\varepsilon} \overrightarrow{2} u \text { in } L^{p}\left(\mathbb{R}^{N} \times \mathcal{Y}\right) \Rightarrow u_{\varepsilon} \rightarrow \bar{u}:=\int_{\mathcal{Y}} u(\cdot, y) d y \text { in } L^{p}\left(\mathbb{R}^{N}\right) \text {. }
\end{aligned}
$$




\section{Two-scale Fourier transform}

In this section we define a two-scale Fourier transform in $L^{1}$ - and $L^{2}$-spaces, and extend to it some properties of the ordinary (single-scale) Fourier transform.

Let us first display the definition of the classical Fourier transform for complexvalued functions of one and two (vector) variables, respectively,

$$
\begin{gathered}
\hat{v}(\zeta)=\left[\mathcal{F}_{1}(v)\right](\zeta):=\int_{\mathbb{R}^{N}} v(x) \exp \{-2 \pi i x \cdot \zeta\} d x \\
\forall \zeta \in \mathbb{R}^{N}, \forall v \in L^{1}\left(\mathbb{R}^{N}\right), \\
\hat{v}(\xi, \mathcal{M})=\left[\mathcal{F}_{2}(v)\right](\xi, \mathcal{M}):=\iint_{\mathbb{R}^{N} \times \mathcal{Y}} v(x, y) \exp \{-2 \pi i(x \cdot \xi+y \cdot \mathcal{M})\} d x d y \\
\forall(\xi, \mathcal{M}) \in \mathbb{R}^{N} \times \mathbb{Z}^{N}, \forall v \in L^{1}\left(\mathbb{R}^{N} \times \mathcal{Y}\right) .
\end{gathered}
$$

We shall refer to $\mathcal{F}_{1}$ and $\mathcal{F}_{2}$ as the single-scale and two-scale Fourier transforms, respectively. Consistently with the theory of Fourier series, in the latter case the second variable is confined to $\mathbb{Z}^{N}$, for $v(x, \cdot)$ is just defined in $\mathcal{Y}$ (the $N$-dimensional unit torus). The reader will also notice that for any $(\xi, \mathcal{M}) \in \mathbb{R}^{N} \times \mathbb{Z}^{N}$ the function $\psi(x, y):=\exp \{-2 \pi i(x \cdot \xi+y \cdot \mathcal{M})\}$ has the properties of test functions of the definition of two-scale convergence, $c f$. (1.1).

It is well known that the operator $\mathcal{F}_{1}$ maps $L^{1}\left(\mathbb{R}^{N}\right)$ to $C_{b}^{0}\left(\mathbb{R}^{N}\right)$ (the space of bounded and continuous functions $\mathbb{R}^{N} \rightarrow \mathbb{C}$ ) continuously. Moreover by density $\mathcal{F}_{1}$ induces a surjective isometry from $L^{2}\left(\mathbb{R}^{N}\right)$ to itself. Similarly, equipping $\mathbb{Z}^{N}$ with the discrete topology and with the counting measure, $\mathcal{F}_{2}$ maps $L^{1}\left(\mathbb{R}^{N} \times \mathcal{Y}\right)$ to $C_{b}^{0}\left(\mathbb{R}^{N} \times \mathbb{Z}^{N}\right)$ continuously, and induces a surjective isometry $L^{2}\left(\mathbb{R}^{N} \times \mathcal{Y}\right) \rightarrow$ $L^{2}\left(\mathbb{R}^{N} \times \mathbb{Z}^{N}\right)$. On $L^{1} \cap L^{2}$ we shall identify the $L^{1}$ - and $L^{2}$-transforms.

Let us now fix any $\varepsilon>0$. For any $\zeta \in \mathbb{R}^{N}$, setting $\mathcal{M}:=\mathcal{N}(\zeta / \varepsilon) \in \mathbb{Z}^{N}$ and $\eta:=\mathcal{R}(\zeta / \varepsilon) \in \mathcal{Y}, c f$. (2.1), we have $\zeta=\varepsilon \mathcal{M}+\varepsilon \eta$. Moreover $\zeta$ spans the whole $\mathbb{R}^{N}$ as $(\eta, \mathcal{M})$ ranges through $\mathcal{Y} \times \mathbb{Z}^{N}$. The definition (3.1) also reads

$$
\begin{aligned}
{\left[\mathcal{F}_{1}(v)\right](\mathcal{M} / \varepsilon+\eta / \varepsilon)=} & \int_{\mathbb{R}^{N}} v(x) \exp \{-2 \pi i(x \cdot \eta / \varepsilon+x \cdot \mathcal{M} / \varepsilon)\} d x \\
& \forall(\eta, \mathcal{M}) \in \mathcal{Y} \times \mathbb{Z}^{N}, \forall v \in L^{1}\left(\mathbb{R}^{N}\right) .
\end{aligned}
$$

Here a further change of variable is in order. Let us set $\xi=\eta / \varepsilon, Y_{1 / \varepsilon}:=\left[0,1 / \varepsilon\left[{ }^{N}\right.\right.$ and denote the corresponding rescaled unit torus by $\mathcal{Y}_{1 / \varepsilon}$. For any $\varepsilon>0$ and any $v \in L^{1}\left(\mathbb{R}^{N}\right)$ let us also define the approximate two-scale Fourier transform (this denomination is justified by Propositions 3.2 and 3.3 below)

$$
\begin{aligned}
{\left[\mathcal{F}_{\varepsilon}(v)\right](\xi, \mathcal{M}) } & :=\int_{\mathbb{R}^{N}} v(x) \exp \{-2 \pi i(x \cdot \xi+x \cdot \mathcal{M} / \varepsilon)\} d x \\
\left(=\left[\mathcal{F}_{1}(v)\right](\mathcal{M} / \varepsilon+\xi)\right) & \forall(\xi, \mathcal{M}) \in \mathcal{Y}_{1 / \varepsilon} \times \mathbb{Z}^{N}, \\
& {\left[\mathcal{F}_{\varepsilon}(v)\right](\xi, \mathcal{M}):=0 \quad \forall(\xi, \mathcal{M}) \in\left(\mathbb{R}^{N} \backslash \mathcal{Y}_{1 / \varepsilon}\right) \times \mathbb{Z}^{N} }
\end{aligned}
$$


Thus by Lemma 2.1, defining $S_{\varepsilon}$ as in (2.2) and noticing that $\exp \{-2 \pi i \mathcal{N} \cdot \mathcal{M}\}=0$ for any $\mathcal{N}, \mathcal{M} \in \mathbb{Z}^{N}$,

$$
\begin{array}{r}
{\left[\mathcal{F}_{\varepsilon}(v)\right](\xi, \mathcal{M}):=\iint_{\mathbb{R}^{N} \times \mathcal{Y}} v\left(S_{\varepsilon}(x, y)\right) \exp \left\{-2 \pi i\left[S_{\varepsilon}(x, y) \cdot \xi+y \cdot \mathcal{M}\right]\right\} d x d y} \\
\forall(\xi, \mathcal{M}) \in \mathcal{Y}_{1 / \varepsilon} \times \mathbb{Z}^{N} .
\end{array}
$$

The operator $\mathcal{F}_{\varepsilon}$ clearly maps $L^{1}\left(\mathbb{R}^{N}\right)$ to $C_{b}^{0}\left(\mathcal{Y}_{1 / \varepsilon} \times \mathbb{Z}^{N}\right)$ continuously. By the next statement $\mathcal{F}_{\varepsilon}$ also induces a surjective isometry $L^{2}\left(\mathbb{R}^{N}\right) \rightarrow L^{2}\left(\mathcal{Y}_{1 / \varepsilon} \times \mathbb{Z}^{N}\right)$ that we shall identify with $\mathcal{F}_{\varepsilon}$ itself. (By the Riesz-Thorin theorem of interpolation of $L^{p}$ spaces, $\mathcal{F}_{\varepsilon}$ also induces a continuous operator $L^{p}\left(\mathbb{R}^{N}\right) \rightarrow L^{p /(p-1)}\left(\mathcal{Y}_{1 / \varepsilon} \times \mathbb{Z}^{N}\right)$ for any $p \in] 1,2[$; here however we shall not address this issue.)

Proposition 3.1. For any $\varepsilon>0$ and any $v \in L^{1}\left(\mathbb{R}^{N}\right)$,

$$
\left\|\mathcal{F}_{\varepsilon}(v)\right\|_{L^{\infty}\left(\mathbb{R}^{N} \times \mathbb{Z}^{N}\right)}=\left\|\mathcal{F}_{1}(v)\right\|_{L^{\infty}\left(\mathbb{R}^{N}\right)}\left(\leq\|v\|_{L^{1}\left(\mathbb{R}^{N}\right)}\right) .
$$

For any $v \in L^{2}\left(\mathbb{R}^{N}\right)$,

$$
\left\|\mathcal{F}_{\varepsilon}(v)\right\|_{L^{2}\left(\mathbb{R}^{N} \times \mathbb{Z}^{N}\right)}=\left\|\mathcal{F}_{1}(v)\right\|_{L^{2}\left(\mathbb{R}^{N}\right)}\left(=\|v\|_{L^{2}\left(\mathbb{R}^{N}\right)}\right) .
$$

Proof. The first statement is obvious. Let us come to the second one. Using Lemma 2.1, setting $\zeta=\varepsilon \mathcal{M}+\varepsilon \eta$, and changing the integration variable twice, for any $v \in L^{2}\left(\mathbb{R}^{N}\right)$ we have

$$
\begin{aligned}
\left\|\mathcal{F}_{\varepsilon}(v)\right\|_{L^{2}\left(\mathbb{R}^{N} \times \mathbb{Z}^{N}\right)}^{2} & =\sum_{\mathcal{M} \in \mathbb{Z}^{N}} \int_{\mathcal{Y}_{1 / \varepsilon}}\left|\left[\mathcal{F}_{1}(v)\right](\mathcal{M} / \varepsilon+\xi)\right|^{2} d \xi \\
& =\varepsilon^{-N} \sum_{\mathcal{M} \in \mathbb{Z}^{N}} \int_{\mathcal{Y}}\left|\left[\mathcal{F}_{1}(v)\right](\mathcal{M} / \varepsilon+\eta / \varepsilon)\right|^{2} d \eta \\
& =\varepsilon^{-N} \int_{\mathbb{R}^{N}}\left|\left[\mathcal{F}_{1}(v)\right](\zeta / \varepsilon)\right|^{2} d \zeta=\left\|\mathcal{F}_{1}(v)\right\|_{L^{2}\left(\mathbb{R}^{N}\right)}^{2} .
\end{aligned}
$$

Next we study the two-scale asymptotic behaviour of the sequence of operators $\left\{\mathcal{F}_{\varepsilon}\right\}$. As $S_{\varepsilon}(x, y) \rightarrow x$ uniformly in $\mathbb{R}^{N} \times \mathcal{Y}$ and $v \circ S_{\varepsilon} \rightarrow v$ whenever $v \overrightarrow{2} v$, by (3.5) we may expect that $\mathcal{F}_{\varepsilon} \rightarrow \mathcal{F}_{2}$ in some sense. This guess is confirmed by the next two statements.

Proposition 3.2. For any sequence $\left\{u_{\varepsilon}\right\}$ of $L^{1}\left(\mathbb{R}^{N}\right)$,

(i) if $u_{\varepsilon} \overrightarrow{2}$ u in $L^{1}\left(\mathbb{R}^{N} \times \mathcal{Y}\right)$ then $\mathcal{F}_{\varepsilon}\left(u_{\varepsilon}\right) \rightarrow \mathcal{F}_{2}(u)$ pointwise in $\mathbb{R}^{N} \times \mathbb{Z}^{N}$;

(ii) if $u_{\varepsilon} \rightarrow \underset{2}{ } u$ in $L^{1}\left(\mathbb{R}^{N} \times \mathcal{Y}\right)$ then $\mathcal{F}_{\varepsilon}\left(u_{\varepsilon}\right) \rightarrow \mathcal{F}_{2}(u)$ uniformly in $A \times \mathbb{Z}^{N}$, for any bounded measurable subset $A$ of $\mathbb{R}^{N}$. 
Proof. (i) By the above definition of weak two-scale convergence, $u_{\varepsilon} \rightarrow 2 \quad u$ in $L^{1}\left(\mathbb{R}^{N} \times \mathcal{Y}\right)$ is tantamount to $u_{\varepsilon}(\varepsilon \mathcal{N}(x / \varepsilon)+\varepsilon y) \rightarrow u(x, y)$ in $L^{1}\left(\mathbb{R}^{N} \times \mathcal{Y}\right)$. As

$$
|[\varepsilon \mathcal{N}(x / \varepsilon)-x] \cdot \xi+\varepsilon y \cdot \xi| \leq 2 \varepsilon|\xi| \quad \forall x, \xi \in \mathbb{R}^{N}, \forall y \in \mathcal{Y},
$$

we then have

$$
\begin{aligned}
w_{\xi, \varepsilon}(x, y) & :=u_{\varepsilon}(\varepsilon \mathcal{N}(x / \varepsilon)+\varepsilon y) \exp \{-2 \pi i([\varepsilon \mathcal{N}(x / \varepsilon)-x] \cdot \xi+\varepsilon y \cdot \xi)\} \\
& \rightarrow u(x, y) \quad \text { in } L^{1}\left(\mathbb{R}^{N} \times \mathcal{Y}\right), \forall \xi \in Y_{1 / \varepsilon} .
\end{aligned}
$$

Therefore, for any $(\xi, \mathcal{M}) \in \mathcal{Y}_{1 / \varepsilon} \times \mathbb{Z}^{N}$, by Lemma 2.1

$$
\begin{aligned}
& {\left[\mathcal{F}_{\varepsilon}\left(u_{\varepsilon}\right)\right](\xi, \mathcal{M})} \\
& =\iint_{\mathbb{R}^{N} \times \mathcal{Y}} u_{\varepsilon}(\varepsilon \mathcal{N}(x / \varepsilon)+\varepsilon y) \exp \{-2 \pi i(\varepsilon \mathcal{N}(x / \varepsilon) \cdot \xi+\varepsilon y \cdot \xi+y \cdot \mathcal{M})\} d x d y \\
& =\iint_{\mathbb{R}^{N} \times \mathcal{Y}} w_{\xi, \varepsilon}(x, y) \exp \{-2 \pi i(x \cdot \xi+y \cdot \mathcal{M})\} d x d y \\
& \rightarrow \iint_{\mathbb{R}^{N} \times \mathcal{Y}} u(x, y) \exp \{-2 \pi i(x \cdot \xi+y \cdot \mathcal{M})\} d x d y=\left[\mathcal{F}_{2}(u)\right](\xi, \mathcal{M}) .
\end{aligned}
$$

We first selected any $\varepsilon$, then any $\xi \in Y_{1 / \varepsilon}$, and then passed to the limit as $\varepsilon \rightarrow 0$. In this limit we might keep $\xi$ fixed, for the family $\left\{Y_{1 / \varepsilon}\right\}$ increases as $\varepsilon$ decreases. As $\mathbb{R}^{N}=\bigcup_{\varepsilon} Y_{1 / \varepsilon},(3.9)$ thus applies to any $\xi \in \mathbb{R}^{N}$.

(ii) If $u_{\varepsilon} \underset{2}{\rightarrow} u$ in $L^{1}\left(\mathbb{R}^{N} \times \mathcal{Y}\right)$ then, for any bounded measurable subset $A$ of $\mathbb{R}^{N}$,

$$
w_{\xi, \varepsilon} \rightarrow u \quad \text { in } L^{1}\left(\mathbb{R}^{N} \times \mathcal{Y}\right), \text { uniformly for } \xi \in A \text {. }
$$

The convergence (3.9) is then uniform in $A \times \mathbb{Z}^{N}$.

Proposition 3.3. For any sequence $\left\{u_{\varepsilon}\right\}$ of $L^{2}\left(\mathbb{R}^{N}\right)$, if $u_{\varepsilon} \underset{2}{\rightarrow} u$ in $L^{2}\left(\mathbb{R}^{N} \times \mathcal{Y}\right)$ then $\mathcal{F}_{\varepsilon}\left(u_{\varepsilon}\right) \rightarrow \mathcal{F}_{2}(u)$ in $L^{2}\left(\mathbb{R}^{N} \times \mathbb{Z}^{N}\right)$.

Proof. By definition of two-scale convergence, $u_{\varepsilon} \rightarrow \quad u$ in $L^{2}\left(\mathbb{R}^{N} \times \mathcal{Y}\right)$ is tantamount to

$$
\tilde{u}_{\varepsilon}(x, y):=u_{\varepsilon}(\varepsilon \mathcal{N}(x / \varepsilon)+\varepsilon y) \rightarrow u(x, y) \quad \text { in } L^{2}\left(\mathbb{R}^{N} \times \mathcal{Y}\right) .
$$

For any compactly supported $\varphi \in L^{2}\left(\mathbb{R}^{N} \times \mathbb{Z}^{N}\right)$, by (3.8)

$$
\begin{aligned}
\psi_{\varepsilon}(\xi, \mathcal{M}, x, y) & :=\varphi(\xi, \mathcal{M}) \exp \{-2 \pi i([\varepsilon \mathcal{N}(x / \varepsilon)-x] \cdot \xi+\varepsilon y \cdot \xi)\} \\
& \rightarrow \varphi(\xi, \mathcal{M}) \quad \text { uniformly in } \mathbb{R}^{N} \times \mathbb{Z}^{N} \times \mathbb{R}^{N} \times \mathcal{Y}
\end{aligned}
$$

With no loss of generality we may assume that $u_{\varepsilon} \in L^{2}\left(\mathbb{R}^{N}\right) \cap L^{1}\left(\mathbb{R}^{N}\right)$ for any $\varepsilon$, so that $\tilde{u}_{\varepsilon} \in L^{2}\left(\mathbb{R}^{N} \times \mathcal{Y}\right) \cap L^{1}\left(\mathbb{R}^{N} \times \mathcal{Y}\right)$. By (3.5), (3.9), (3.11) and by the weak 
continuity of $\mathcal{F}_{2}$ in $L^{2}\left(\mathbb{R}^{N} \times \mathbb{Z}^{N}\right)$, we then have (understanding the integral with respect to $(x, y)$ in the sense of Cauchy's principal value)

$$
\begin{aligned}
& \lim _{\varepsilon \rightarrow 0} \sum_{m \in \mathbb{Z}^{N}} \int_{\mathbb{R}^{N}}\left[\mathcal{F}_{\varepsilon}\left(u_{\varepsilon}\right)\right](\xi, \mathcal{M}) \varphi(\xi, \mathcal{M}) d \xi \\
& =\lim _{\varepsilon \rightarrow 0} \sum_{m \in \mathbb{Z}^{N}} \int_{\mathbb{R}^{N}} d \xi \iint_{\mathbb{R}^{N} \times \mathcal{Y}} u_{\varepsilon}(\varepsilon \mathcal{N}(x / \varepsilon)+\varepsilon y) \\
& =\lim _{\varepsilon \rightarrow 0} \sum_{m \in \mathbb{Z}^{N}} \int_{\mathbb{R}^{N}} d \xi \iint_{\mathbb{R}^{N} \times \mathcal{Y}} \tilde{u}_{\varepsilon}(x, y) \exp \{-2 \pi i(x \cdot \xi+y \cdot \mathcal{M})\} \psi_{\varepsilon}(\xi, \mathcal{M}, x, y) d x d y \\
& =\lim _{\varepsilon \rightarrow 0} \sum_{m \in \mathbb{Z}^{N}} \int_{\mathbb{R}^{N}} d \xi \iint_{\mathbb{R}^{N} \times \mathcal{Y}} \tilde{u}_{\varepsilon}(x, y) \exp \{-2 \pi i(x \cdot \xi+y \cdot \mathcal{M})\} \varphi(\xi, \mathcal{M}) d x d y \\
& =\lim _{\varepsilon \rightarrow 0} \sum_{m \in \mathbb{Z}^{N}} \int_{\mathbb{R}^{N}}\left[\mathcal{F}_{2}\left(\tilde{u}_{\varepsilon}\right)\right](\xi, \mathcal{M}) \varphi(\xi, \mathcal{M}) d \xi \\
& =\sum_{m \in \mathbb{Z}^{N}} \int_{\mathbb{R}^{N}}\left[\mathcal{F}_{2}(u)\right](\xi, \mathcal{M}) \varphi(\xi, \mathcal{M}) d \xi .
\end{aligned}
$$

As the compactly supported functions $\varphi$ are dense in $L^{2}\left(\mathbb{R}^{N} \times \mathbb{Z}^{N}\right)$, we then conclude that $\mathcal{F}_{\varepsilon}\left(u_{\varepsilon}\right) \rightarrow \mathcal{F}_{2}(u)$ in this space.

The definitions of the Fourier transform and the results of this section are trivially extended to vector-valued functions $\mathbb{R}^{N} \rightarrow \mathbb{C}^{M}$ for any positive integers $M, N$.

\section{Two-scale Fourier transform of differential operators}

In this section we extend to the two-scale Fourier transform some properties of the ordinary Fourier transform of differential operators. First let us set

$$
\tilde{Y}:=\left[-1 / 2,1 / 2\left[^{N}, \quad \tilde{Y}_{1 / \varepsilon}:=\left[-1 /(2 \varepsilon), 1 /(2 \varepsilon)\left[{ }^{N},\right.\right.\right.\right.
$$

denote by $\tilde{\mathcal{Y}}$ and $\tilde{\mathcal{Y}}_{1 / \varepsilon}$ the corresponding tori, and redefine $\mathcal{F}_{\varepsilon}$ by replacing $\mathcal{Y}_{1 / \varepsilon}$ with $\tilde{\mathcal{Y}}_{1 / \varepsilon}$ in (3.4). Let us also set

$$
\begin{gathered}
a(\lambda):=\frac{\exp \{i \lambda\}-1}{i \lambda} \quad \forall \lambda \in[-\pi, \pi[, \quad a(0):=1, \\
\xi_{[\varepsilon]}:=\xi \prod_{j=1}^{N} a\left(2 \pi \varepsilon \xi_{j}\right) \quad \forall \xi \in \tilde{\mathcal{Y}}_{1 / \varepsilon}, \forall \varepsilon>0 ;
\end{gathered}
$$


thus

$$
\xi_{[\varepsilon]}=\xi \prod_{j=1}^{N} \frac{\exp \left\{2 \pi i \varepsilon \xi_{j}\right\}-1}{2 \pi i \varepsilon \xi_{j}} \quad \text { if } \xi_{j} \neq 0 \text { for any } j, \quad \xi_{[\varepsilon]}=0 \quad \text { otherwise. }
$$

An elementary calculation shows that $\frac{2}{\pi} \leq|a(\lambda)| \leq 1$ for any $\lambda \in[-\pi, \pi[$; hence

$$
\begin{gathered}
\left(\frac{2}{\pi}\right)^{N}|\xi| \leq\left|\xi_{[\varepsilon]}\right| \leq|\xi| \quad \forall \xi \in \tilde{\mathcal{Y}}_{1 / \varepsilon}, \forall \varepsilon>0 ; \\
\xi_{[\varepsilon]} \rightarrow \xi \quad \text { as } \varepsilon \rightarrow 0, \forall \xi \in \mathbb{R} \text { (not uniformly). }
\end{gathered}
$$

\section{Approximate two-scale derivatives}

For $j=1, \ldots, N$, let us denote by $\nabla_{j} \varphi$ the partial derivative with respect to $x_{j}$ of any function $\varphi(x)$, and by $\nabla_{x_{j}} \psi$ and $\nabla_{y_{j}} \psi$ the partial derivatives of any function $\psi(x, y)$. Let us also denote by $e_{j}$ the unit vector of the $x_{j}$-axis, define the shift operator $\left(\tau_{h} v\right)(x):=v(x+h)$ for any $x, h \in \mathbb{R}^{N}$, set

$$
\nabla_{\varepsilon, j}:=\frac{\tau_{\varepsilon e_{j}}-I}{\varepsilon}, \quad \nabla_{\varepsilon}^{\alpha}:=\prod_{j=1}^{N} \nabla_{\varepsilon, j}^{\alpha_{j}}, \quad \nabla^{\alpha}=\prod_{j=1}^{N} \nabla_{j}^{\alpha_{j}} \quad \forall \alpha \in \mathbb{N}^{N}, \forall \varepsilon>0,
$$

and define $\nabla_{-\varepsilon, j}, \nabla_{x}^{\alpha}, \nabla_{y}^{\alpha}$ similarly. The operators $\nabla_{\varepsilon}$ and $\varepsilon \nabla$ may be regarded as approximate two-scale derivatives because of the next result.

Proposition 4.1 ( [31]). Let $p \in] 1,+\infty\left[\right.$ and $\left\{u_{\varepsilon}\right\}$ be a sequence in $L^{p}\left(\mathbb{R}^{N}\right)$ such that $u_{\varepsilon} \underset{2}{ } u$ in $L^{p}\left(\mathbb{R}^{N} \times \mathcal{Y}\right)$.

(i) If $\left\{\nabla_{\varepsilon} u_{\varepsilon}\right\}$ is bounded in $L^{p}\left(\mathbb{R}^{N}\right)^{N}$ then

$$
u \in W^{1, p}\left(\mathbb{R}^{N} ; L^{p}(\mathcal{Y})\right), \quad \nabla_{\varepsilon} u_{\varepsilon} \underset{2}{\rightarrow} \nabla_{x} u \text { in } L^{p}\left(\mathbb{R}^{N} \times \mathcal{Y}\right)^{N} .
$$

(ii) If $\left\{\varepsilon \nabla u_{\varepsilon}\right\}$ is bounded in $L^{p}\left(\mathbb{R}^{N}\right)^{N}$ then

$$
u \in L^{p}\left(\mathbb{R}^{N} ; W^{1, p}(\mathcal{Y})\right), \quad \varepsilon \nabla u_{\varepsilon} \underset{2}{\rightarrow} \nabla_{y} u \quad \text { in } L^{p}\left(\mathbb{R}^{N} \times \mathcal{Y}\right)^{N} .
$$

Next we extend some properties to the two-scale Fourier transform.

Proposition 4.2. For any $v \in L^{1}\left(\mathbb{R}^{N}\right)$ and any $j \in\{1, \ldots, N\}$,

$$
\left[\mathcal{F}_{\varepsilon}\left(\nabla_{\varepsilon, j} v\right)\right](\xi, \mathcal{M})=2 \pi i \xi_{[\varepsilon] j}\left[\mathcal{F}_{\varepsilon}(v)\right](\xi, \mathcal{M}) \quad \forall(\xi, \mathcal{M}) \in \tilde{\mathcal{Y}}_{1 / \varepsilon} \times \mathbb{Z}^{N} .
$$

If $v \in L^{2}\left(\mathbb{R}^{N}\right)$ then the same holds for almost all $\xi \in \tilde{\mathcal{Y}}_{1 / \varepsilon}$ and any $\mathcal{M} \in \mathbb{Z}^{N}$. Moreover, for any sequence $\left\{u_{\varepsilon}\right\}$ of $L^{2}\left(\mathbb{R}^{N}\right)$,

$$
\begin{aligned}
& \left\|\nabla_{\varepsilon, j} u_{\varepsilon}\right\|_{L^{2}\left(\mathbb{R}^{N}\right)} \text { is bounded } \Leftrightarrow \\
& \left\|\xi_{[\varepsilon] j} \mathcal{F}_{\varepsilon}\left(u_{\varepsilon}\right)\right\|_{L^{2}\left(\tilde{\mathcal{Y}}_{1 / \varepsilon} \times \mathbb{Z}^{N}\right)} \text { is bounded } \Leftrightarrow \\
& \left\|\xi_{j} \mathcal{F}_{\varepsilon}\left(u_{\varepsilon}\right)\right\|_{L^{2}\left(\tilde{\mathcal{Y}}_{1 / \varepsilon} \times \mathbb{Z}^{N}\right)} \text { is bounded. }
\end{aligned}
$$


Proof. First note that, defining $S_{\varepsilon}(x, y)$ as in (2.2), for any $\varepsilon>0$

$$
\begin{gathered}
\left(\nabla_{\varepsilon, j} v\right)\left(S_{\varepsilon}(x, y)\right)=\nabla_{\varepsilon, x_{j}}\left[v\left(S_{\varepsilon}(x, y)\right)\right] \quad \forall v \in L^{1}\left(\mathbb{R}^{N}\right), \\
\iint_{\mathbb{R}^{N} \times \mathcal{Y}} \nabla_{\varepsilon, x_{j}}\left[v\left(S_{\varepsilon}(x, y)\right)\right] w\left(S_{\varepsilon}(x, y)\right) d x d y \\
=-\iint_{\mathbb{R}^{N} \times \mathcal{Y}} v\left(S_{\varepsilon}(x, y)\right) \nabla_{-\varepsilon, x_{j}} w\left(S_{\varepsilon}(x, y)\right) d x d y \quad \forall v, w \in L^{2}\left(\mathbb{R}^{N}\right), \\
\nabla_{-\varepsilon, x_{j}} \exp \{-2 \pi i(S(x / \varepsilon) \cdot \xi+y \cdot \mathcal{M})\}=-2 \pi i \xi[\varepsilon] j \exp \{-2 \pi i(S(x / \varepsilon) \cdot \xi+y \cdot \mathcal{M})\} \\
\forall(x, y, \xi, \mathcal{M}) \in \mathbb{R}^{N} \times \mathcal{Y} \times \tilde{Y}_{1 / \varepsilon} \times \mathbb{Z}^{N} .
\end{gathered}
$$

For any $v \in L^{1}\left(\mathbb{R}^{N}\right)$, by (3.5) we then have

$$
\begin{aligned}
& {\left[\mathcal{F}_{\varepsilon}\left(\nabla_{\varepsilon, j} v\right)\right](\xi, \mathcal{M})} \\
& =\iint_{\mathbb{R}^{N} \times \mathcal{Y}} \nabla_{\varepsilon, x_{j}}\left[v\left(S_{\varepsilon}(x, y)\right)\right] \exp \{-2 \pi i(S(x / \varepsilon) \cdot \xi+y \cdot \mathcal{M})\} d x d y \\
& =-\iint_{\mathbb{R}^{N} \times \mathcal{Y}} v\left(S_{\varepsilon}(x, y)\right) \nabla_{-\varepsilon, x_{j}} \exp \{-2 \pi i(S(x / \varepsilon) \cdot \xi+y \cdot \mathcal{M})\} d x d y \\
& =2 \pi i \xi_{[\varepsilon] j} \iint_{\mathbb{R}^{N} \times \mathcal{Y}} v\left(S_{\varepsilon}(x, y)\right) \exp \{-2 \pi i(S(x / \varepsilon) \cdot \xi+y \cdot \mathcal{M})\} d x d y \\
& =2 \pi i \xi_{[\varepsilon] j}\left[\mathcal{F}_{\varepsilon}(v)\right](\xi, \mathcal{M}) \quad \forall(\xi, \mathcal{M}) \in \tilde{\mathcal{Y}}_{1 / \varepsilon} \times \mathbb{Z}^{N} .
\end{aligned}
$$

This yields (4.8), and by Proposition 3.2 the first equivalence of (4.9) follows; the second one stems from (4.3).

Proposition 4.3. For any $v \in W^{1,1}\left(\mathbb{R}^{N}\right)$ and any $j \in\{1, \ldots, N\}$,

$$
\left[\mathcal{F}_{\varepsilon}\left(\varepsilon \nabla_{j} v\right)\right](\xi, \mathcal{M})=2 \pi i\left(\mathcal{M}_{j}+\varepsilon \xi_{j}\right)\left[\mathcal{F}_{\varepsilon}(v)\right](\xi, \mathcal{M}) \quad \forall(\xi, \mathcal{M}) \in \tilde{\mathcal{Y}}_{1 / \varepsilon} \times \mathbb{Z}^{N}
$$

If $v \in H^{1}\left(\mathbb{R}^{N}\right)$ then the same holds for almost all $\xi \in \tilde{\mathcal{Y}}_{1 / \varepsilon}$ and any $\mathcal{M} \in \mathbb{Z}^{N}$. Moreover, for any sequence $\left\{u_{\varepsilon}\right\}$ of $H^{1}\left(\mathbb{R}^{N}\right)$ and any $j \in\{1, \ldots, N\}$,

$\left\|\varepsilon \nabla_{j} u_{\varepsilon}\right\|_{L^{2}\left(\mathbb{R}^{N}\right)} \quad$ is bounded $\Leftrightarrow\left\|\left(\mathcal{M}_{j}+\varepsilon \xi_{j}\right) \mathcal{F}_{\varepsilon}\left(u_{\varepsilon}\right)\right\|_{L^{2}\left(\tilde{\mathcal{Y}}_{1 / \varepsilon} \times \mathbb{Z}^{N}\right)}$ is bounded.

Proof. We have

$$
\begin{aligned}
& {\left[\mathcal{F}_{\varepsilon}\left(\varepsilon \nabla_{j} v\right)\right](\xi, \mathcal{M})=\varepsilon \int_{\mathbb{R}^{N}} \nabla_{j} v(x) \exp \{-2 \pi i x \cdot(\xi+\mathcal{M} / \varepsilon)\} d x} \\
& =-\varepsilon \int_{\mathbb{R}^{N}} v(x) \nabla_{j} \exp \{-2 \pi i x \cdot(\xi+\mathcal{M} / \varepsilon)\} d x \\
& =2 \pi i\left(\varepsilon \xi_{j}+\mathcal{M}_{j}\right) \int_{\mathbb{R}^{N}} v(x) \exp \{-2 \pi i x \cdot(\xi+\mathcal{M} / \varepsilon)\} d x \\
& =2 \pi i\left(\varepsilon \xi_{j}+\mathcal{M}_{j}\right)\left[\mathcal{F}_{\varepsilon}(v)\right](\xi, \mathcal{M}) \quad \forall(\xi, \mathcal{M}) \in \tilde{\mathcal{Y}}_{1 / \varepsilon} \times \mathbb{Z}^{N} ;
\end{aligned}
$$

the equation (4.11) thus holds. By Proposition 3.1, (4.12) then follows. 
The two latter propositions have several consequences, that may be checked by routine procedures.

Corollary 4.4. Let $\left\{u_{\varepsilon}\right\}$ be a sequence of $H^{1}\left(\mathbb{R}^{N}\right)^{N}$. Then

$\left\|\nabla_{\varepsilon} u_{\varepsilon}\right\|_{L^{2}\left(\mathbb{R}^{N}\right)^{N}}$ is bounded $\Leftrightarrow\left\|\xi_{[\varepsilon]} \mathcal{F}_{\varepsilon}\left(u_{\varepsilon}\right)\right\|_{L^{2}\left(\tilde{\mathcal{Y}}_{1 / \varepsilon} \times \mathbb{Z}^{N}\right)^{N}}$ is bounded;

$\left\|\varepsilon \nabla u_{\varepsilon}\right\|_{L^{2}\left(\mathbb{R}^{N}\right)^{N}}$ is bounded $\Leftrightarrow\left\|(\varepsilon \xi+\mathcal{M}) \mathcal{F}_{\varepsilon}\left(u_{\varepsilon}\right)\right\|_{L^{2}\left(\tilde{\mathcal{Y}}_{1 / \varepsilon} \times \mathbb{Z}^{N}\right)^{N}}$ is bounded;

$\left\|\nabla_{\varepsilon} \times u_{\varepsilon}\right\|_{L^{2}\left(\mathbb{R}^{N}\right)^{N^{2}}}$ is bounded $\Leftrightarrow\left\|\xi_{[\varepsilon]} \times \mathcal{F}_{\varepsilon}\left(u_{\varepsilon}\right)\right\|_{L^{2}\left(\tilde{\mathcal{Y}}_{1 / \varepsilon} \times \mathbb{Z}^{N}\right)^{N^{2}}}$ is bounded;

$\left\|\varepsilon \nabla \times u_{\varepsilon}\right\|_{L^{2}\left(\mathbb{R}^{N}\right)^{N^{2}}}$ is bounded $\Leftrightarrow\left\|(\varepsilon \xi+\mathcal{M}) \times \mathcal{F}_{\varepsilon}\left(u_{\varepsilon}\right)\right\|_{L^{2}\left(\tilde{\mathcal{Y}}_{1 / \varepsilon} \times \mathbb{Z}^{N}\right)^{N^{2}}}$ is bounded;

$\left\|\nabla_{\varepsilon} \cdot u_{\varepsilon}\right\|_{L^{2}\left(\mathbb{R}^{N}\right)}$ is bounded $\Leftrightarrow\left\|\xi_{[\varepsilon]} \cdot \mathcal{F}_{\varepsilon}\left(u_{\varepsilon}\right)\right\|_{L^{2}\left(\tilde{\mathcal{Y}}_{1 / \varepsilon} \times \mathbb{Z}^{N}\right)}$ is bounded;

$\left\|\varepsilon \nabla \cdot u_{\varepsilon}\right\|_{L^{2}\left(\mathbb{R}^{N}\right)}$ is bounded $\Leftrightarrow\left\|(\varepsilon \xi+\mathcal{M}) \cdot \mathcal{F}_{\varepsilon}\left(u_{\varepsilon}\right)\right\|_{L^{2}\left(\tilde{\mathcal{Y}}_{1 / \varepsilon} \times \mathbb{Z}^{N}\right)}$ is bounded.

\section{Two-scale div-curl lemma}

In this section we extend the div-curl lemma to two-scale convergence. We still assume that $\Omega$ is a (possibly unbounded) domain of $\mathbb{R}^{N}$, and deal with complexvalued functions. We shall extend to $\mathbb{R}^{N}$ with vanishing value any function defined in $\Omega$, and use the definitions (1.9), (1.13)-(1.15).

Theorem 5.1 (Two-scale div-curl lemma). Let $\left\{u_{\varepsilon}^{(1)}\right\},\left\{u_{\varepsilon}^{(2)}\right\},\left\{w_{\varepsilon}^{(1)}\right\},\left\{w_{\varepsilon}^{(2)}\right\}$ be four sequences of $L_{\mathrm{loc}}^{2}(\Omega)^{N}$ such that $\nabla \times u_{\varepsilon}^{(1)}, \nabla \times u_{\varepsilon}^{(2)} \in L_{\mathrm{loc}}^{2}(\Omega)^{N^{2}}$ and $\nabla \cdot w_{\varepsilon}^{(2)} \in$ $L_{\mathrm{loc}}^{2}(\Omega)$ for any $\varepsilon$. If

$$
\begin{aligned}
u_{\varepsilon}:= & \left(u_{\varepsilon}^{(1)}, u_{\varepsilon}^{(2)}\right) \rightarrow \overrightarrow{2} u:=\left(u^{(1)}, u^{(2)}\right) \\
w_{\varepsilon}:= & \left(w_{\varepsilon}^{(1)}, w_{\varepsilon}^{(2)}\right) \underset{2}{\rightarrow} w:=\left(w^{(1)}, w^{(2)}\right) \\
& \left\{G_{\varepsilon} \times u_{\varepsilon}\right\} \text { is bounded in } L_{\mathrm{loc}}^{2}(\Omega)^{4 N^{2}}, \\
& \left\{G_{\varepsilon} \cdot w_{\varepsilon}\right\} \text { is bounded in } L_{\mathrm{loc}}^{2}(\Omega),
\end{aligned}
$$

then $u_{\varepsilon} \cdot w_{\varepsilon} \frac{*}{2} u \cdot w$ in $C_{c}^{0}(\Omega \times \mathcal{Y})^{\prime}$, that is,

$$
\begin{gathered}
\int_{\Omega}\left[u_{\varepsilon}^{(1)}(x) \cdot w_{\varepsilon}^{(1)}(x)+u_{\varepsilon}^{(2)}(x) \cdot w_{\varepsilon}^{(2)}(x)\right] \varphi(x, x / \varepsilon) d x \\
\rightarrow \iint_{\Omega \times \mathcal{Y}}\left[u^{(1)}(x, y) \cdot w^{(1)}(x, y)+u^{(2)}(x, y) \cdot w^{(2)}(x, y)\right] \varphi(x, y) d x d y \\
\forall \varphi \in C_{c}^{0}(\Omega \times \mathcal{Y}) .
\end{gathered}
$$


Notice that, by Proposition 4.1, (5.2) and (5.3) respectively entail that

$$
G \times u \in L_{\mathrm{loc}}^{2}(\Omega \times \mathcal{Y})^{4 N^{2}}, \quad G \cdot w \in L_{\mathrm{loc}}^{2}(\Omega \times \mathcal{Y}),
$$

and the latter scalar equals $\nabla_{x} \cdot w^{(1)}+\nabla_{y} \cdot w^{(2)}=\sum_{i=0}^{N}\left(\nabla_{x_{i}} \cdot w_{i}^{(1)}+\nabla_{y_{i}} \cdot w_{i}^{(2)}\right)$.

Proof. We follow the lines of the argument of Theorem 1 of [18], but here the approximate two-scale transform $\mathcal{F}_{\varepsilon}$ is used aside the two-scale Fourier transform $\mathcal{F}_{2}, c f$. (3.2) and (3.4). We split the proof into three parts.

(i) Let us fix any $\varphi, \psi \in \mathcal{D}(\Omega \times \mathcal{Y})$ such that $\psi \equiv 1$ on the support of $\varphi$ (so that $\psi \varphi \equiv \varphi)$, set

$$
\begin{aligned}
U_{\varepsilon}(x) & :=\left(u_{\varepsilon}^{(1)}(x), u_{\varepsilon}^{(2)}(x)\right) \varphi(x, x / \varepsilon) \\
W_{\varepsilon}(x) & :=\left(w_{\varepsilon}^{(1)}(x), w_{\varepsilon}^{(2)}(x)\right) \psi(x, x / \varepsilon) \\
U(x, y) & :=\left(u^{(1)}(x, y), u^{(2)}(x, y)\right) \varphi(x, y) \quad \forall(x, y) \in \Omega \times \mathcal{Y}, \forall \varepsilon, \\
W(x, y) & :=\left(w^{(1)}(x, y), w^{(2)}(x, y)\right) \psi(x, y)
\end{aligned}
$$

and extend these functions to $\mathbb{R}^{N}\left(\mathbb{R}^{N} \times \mathcal{Y}\right.$, respectively) with vanishing value. Thus

$$
U_{\varepsilon}, W_{\varepsilon} \in L^{1}\left(\mathbb{R}^{N}\right)^{2 N} \cap L^{2}\left(\mathbb{R}^{N}\right)^{2 N}, \quad U, W \in L^{1}\left(\mathbb{R}^{N} \times \mathcal{Y}\right)^{2 N} \cap L^{2}\left(\mathbb{R}^{N} \times \mathcal{Y}\right)^{2 N},
$$

so that we may set

$$
\hat{U}_{\varepsilon}:=\mathcal{F}_{\varepsilon}\left(U_{\varepsilon}\right), \quad \hat{W}_{\varepsilon}:=\mathcal{F}_{\varepsilon}\left(W_{\varepsilon}\right), \quad \hat{U}:=\mathcal{F}_{2}(U), \quad \hat{W}:=\mathcal{F}_{2}(W) \quad \forall \varepsilon .
$$

As these functions take values in $\mathbb{R}^{2 N}$, these transforms are defined componentwise.

As the functions $U_{\varepsilon}$ and $W_{\varepsilon}$ are uniformly bounded in $L^{1}\left(\mathbb{R}^{N}\right)^{2 N} \cap L^{2}\left(\mathbb{R}^{N}\right)^{2 N}$ with respect to $\varepsilon$, by Proposition 3.1 we infer that

$$
\hat{U}_{\varepsilon}, \hat{W}_{\varepsilon} \text { are uniformly bounded in } L^{\infty}\left(\mathbb{R}^{N} \times \mathbb{Z}^{N}\right)^{2 N} \cap L^{2}\left(\mathbb{R}^{N} \times \mathbb{Z}^{N}\right)^{2 N} \text {. }
$$

By (5.1)

$$
U_{\varepsilon} \underset{2}{\rightarrow} U, \quad W_{\varepsilon} \overrightarrow{2} W \quad \text { in } L^{1}\left(\mathbb{R}^{N} \times \mathcal{Y}\right)^{2 N} \cap L^{2}\left(\mathbb{R}^{N} \times \mathcal{Y}\right)^{2 N} ;
$$

by Propositions 3.2 and 3.3 it then follows that

$$
\begin{array}{ll}
\hat{U}_{\varepsilon} \rightarrow \hat{U}, \quad \hat{W}_{\varepsilon} \rightarrow \hat{W} & \text { pointwise in } \mathbb{R}^{N} \times \mathbb{Z}^{N}, \\
\hat{U}_{\varepsilon} \rightarrow \hat{U}, \quad \hat{W}_{\varepsilon} \rightarrow \hat{W} & \text { in } L^{2}\left(\mathbb{R}^{N} \times \mathbb{Z}^{N}\right)^{2 N} .
\end{array}
$$

Hence $\hat{U}_{\varepsilon} \cdot \hat{W}_{\varepsilon} \rightarrow \hat{U} \cdot \hat{W}$ pointwise in $\mathbb{R}^{N} \times \mathbb{Z}^{N}$, and this sequence is bounded in $L^{\infty}\left(\mathbb{R}^{N} \times \mathbb{Z}^{N}\right)$; by the Lebesgue dominated convergence theorem we then infer that (omitting restrictions)

$\hat{U}_{\varepsilon} \cdot \hat{W}_{\varepsilon} \rightarrow \hat{U} \cdot \hat{W} \quad$ in $L^{1}(C)$, for any bounded measurable set $C \subset \mathbb{R}^{N} \times \mathbb{Z}^{N}$. 
(ii) Let us define $\xi_{[\varepsilon]}$ as in (4.2) and set

$$
\zeta_{\varepsilon}(\xi, \mathcal{M}):=\left(\xi_{[\varepsilon]}, \mathcal{M}+\varepsilon \xi\right)\left(\in \mathbb{C}^{2 N}\right) \quad \forall(\xi, \mathcal{M}) \in \tilde{\mathcal{Y}}_{1 / \varepsilon} \times \mathbb{Z}^{N} .
$$

By (5.2), (5.3) and Corollary 4.4,

$$
\hat{U}_{\varepsilon}, \hat{W}_{\varepsilon}, \zeta_{\varepsilon} \times \hat{U}_{\varepsilon}, \zeta_{\varepsilon} \cdot \hat{W}_{\varepsilon} \text { are uniformly bounded in } L^{2}\left(\mathbb{R}^{N} \times \mathbb{Z}^{N}\right)^{M} ;
$$

here either $M=1$ or $M=2 N$ or $M=4 N^{2}$ depending on the case. By the elementary identity

$$
\begin{array}{r}
\lambda_{k} w \cdot u=\lambda_{k} \sum_{j=1}^{2 N} w_{j} u_{j}=\left(\sum_{j=1}^{2 N} \lambda_{j} w_{j}\right) u_{k}+\sum_{j=1}^{2 N} w_{j}\left(\lambda_{k} u_{j}-\lambda_{j} u_{k}\right) \\
\quad \text { for } k=1, \ldots, 2 N, \forall w, u, \lambda \in \mathbb{C}^{2 N}
\end{array}
$$

(namely $\lambda w \cdot u=(\lambda \cdot w) u+w(\lambda \times u)$ ), taking $w=\hat{W}_{\varepsilon}, u=\hat{U}_{\varepsilon}$ and $\lambda=\zeta_{\varepsilon}(\xi, \mathcal{M})$, we thus get

$$
\left|\zeta_{\varepsilon} \hat{W}_{\varepsilon} \cdot \hat{U}_{\varepsilon}\right| \leq\left|\zeta_{\varepsilon} \cdot \hat{W}_{\varepsilon}\right|\left|\hat{U}_{\varepsilon}\right|+\left|\hat{W}_{\varepsilon}\right|\left|\zeta_{\varepsilon} \times \hat{U}_{\varepsilon}\right| \quad \text { in } \mathbb{R}^{N} \times \mathbb{Z}^{N}, \forall \varepsilon
$$

Here $\zeta_{\varepsilon} \hat{W}_{\varepsilon} \cdot \hat{U}_{\varepsilon} \in \mathbb{C}^{2 N}$ and this is a function of $(\xi, \mathcal{M}) \in \mathbb{R}^{N} \times \mathbb{Z}^{N}$; moreover $\zeta_{\varepsilon} \cdot \hat{W}_{\varepsilon} \in \mathbb{C}$ and $\zeta_{\varepsilon} \times \hat{U}_{\varepsilon} \in \mathbb{C}^{4 N^{2}}$. By (5.11) and by the latter inequality we infer that

$$
\left\|W_{\varepsilon} \cdot \hat{U}_{\varepsilon}\right\|_{L^{1}\left(\mathbb{R}^{N} \times \mathbb{Z}^{N}\right)^{2 N}},\left\|\zeta_{\varepsilon} W_{\varepsilon} \cdot \hat{U}_{\varepsilon}\right\|_{L^{1}\left(\mathbb{R}^{N} \times \mathbb{Z}^{N}\right)^{2 N}} \leq \text { Constant. }
$$

(iii) By (5.9) and (5.13) we get

$$
\hat{W}_{\varepsilon} \cdot \hat{U}_{\varepsilon} \rightarrow \hat{W} \cdot \hat{U} \quad \text { in } L^{1}\left(\mathbb{R}^{N} \times \mathbb{Z}^{N}\right),
$$

whence

$$
\sum_{\mathcal{M} \in \mathbb{Z}^{N}} \int_{\mathbb{R}^{N}} \hat{W}_{\varepsilon}(\xi, \mathcal{M}) \cdot \hat{U}_{\varepsilon}(\xi, \mathcal{M}) d \xi \rightarrow \sum_{\mathcal{M} \in \mathbb{Z}^{N}} \int_{\mathbb{R}^{N}} \hat{W}(\xi, \mathcal{M}) \cdot \hat{U}(\xi, \mathcal{M}) d \xi
$$

By the $L^{2}$-isometry of both the approximate and ordinary two-scale Fourier transforms, $c f$. Proposition 3.1, we have

$$
\begin{aligned}
& \sum_{\mathcal{M} \in \mathbb{Z}^{N}} \int_{\mathbb{R}^{N}} \hat{W}_{\varepsilon}(\xi, \mathcal{M}) \cdot \hat{U}_{\varepsilon}(\xi, \mathcal{M}) d \xi=\int_{\mathbb{R}^{N}} W_{\varepsilon}(x) \cdot U_{\varepsilon}(x) d x \quad \forall \varepsilon \\
& \sum_{\mathcal{M} \in \mathbb{Z}^{N}} \int_{\mathbb{R}^{N}} \hat{W}(\xi, \mathcal{M}) \cdot \hat{U}(\xi, \mathcal{M}) d \xi=\iint_{\mathbb{R}^{N} \times \mathcal{Y}} W(x, y) \cdot U(x, y) d x d y .
\end{aligned}
$$


Recalling (5.6), the convergence (5.15) then also reads

$$
\begin{aligned}
& \int_{\Omega} u_{\varepsilon}(x) \cdot w_{\varepsilon}(x) \varphi(x, x / \varepsilon) d x=\int_{\mathbb{R}^{N}} U_{\varepsilon}(x) \cdot W_{\varepsilon}(x) d x \\
& \rightarrow \iint_{\mathbb{R}^{N} \times \mathcal{Y}} U(x, y) \cdot W(x, y) d x d y=\iint_{\mathbb{R}^{N} \times \mathcal{Y}} u(x, y) \cdot w(x, y) \varphi(x, y) d x d y .
\end{aligned}
$$

Thus (5.4) holds for any $\varphi \in \mathcal{D}(\Omega \times \mathcal{Y})$; as this space is dense in $C_{c}^{0}(\Omega \times \mathcal{Y})$ we get the thesis.

As we pointed out in the introduction, the hypothesis (1.2) of Theorem 1 may be replaced by the weaker condition (1.5). An analogous statement applies to the two-scale framework.

Proposition 5.2. Theorem 5.1 holds also if the hypotheses (5.2) and (5.3) are replaced by

$$
\begin{aligned}
& \left\{G_{\varepsilon} \times\left(u_{\varepsilon} \circ S_{\varepsilon}\right)\right\} \text { is in a compact subset of } H^{-1}(\Omega \times \mathcal{Y})^{4 N^{2}}, \\
& \left\{G_{\varepsilon} \cdot\left(w_{\varepsilon} \circ S_{\varepsilon}\right)\right\} \text { is in a compact subset of } H^{-1}(\Omega \times \mathcal{Y}) .
\end{aligned}
$$

(Admittedly these hypotheses are somehow indirect.)

Proof. First, for any $R>0$, let us denote by $B_{R}$ the ball of $\mathbb{R}^{N} \times \mathbb{Z}^{N}$ with center the origin and radius $R$, and by $B_{R}^{\prime}$ its complementary set. Here it suffices to modify part (ii) of the argument of Theorem 5.1 as follows. By (4.8), (4.11), (5.16) and (5.17),

$$
\begin{aligned}
& \left\|\frac{\left(\xi_{[\varepsilon]}, \mathcal{M}+\varepsilon \xi\right) \times \hat{U}_{\varepsilon}}{1+\left|\xi_{[\varepsilon]}\right|+|\mathcal{M}+\varepsilon \xi|}\right\|_{L^{2}\left(B_{R}^{\prime}\right)} \rightarrow 0 \text { as } R \rightarrow+\infty, \text { uniformly with respect to } \varepsilon, \\
& \left\|\frac{\left(\xi_{[\varepsilon]}, \mathcal{M}+\varepsilon \xi\right) \cdot \hat{W}_{\varepsilon}}{1+\left|\xi_{[\varepsilon]}\right|+|\mathcal{M}+\varepsilon \xi|}\right\|_{L^{2}\left(B_{R}^{\prime}\right)} \rightarrow 0 \text { as } R \rightarrow+\infty \text {, uniformly with respect to } \varepsilon .
\end{aligned}
$$

In place of (5.13) here we can then just state the weaker property

$$
\left\|\hat{U}_{\varepsilon} \cdot \hat{W}_{\varepsilon}\right\|_{L^{1}\left(B_{R}^{\prime}\right)} \rightarrow 0 \quad \text { as } R \rightarrow+\infty, \text { uniformly with respect to } \varepsilon ;
$$

after (5.9) this however suffices to entail (5.14). The remainder of the proof holds unchanged.

The next result applies to scalar functions. 
Proposition 5.3. Let $\left\{u_{\varepsilon}\right\}$ be a sequence of $L_{\mathrm{loc}}^{2}(\Omega)$ and $\left\{w_{\varepsilon}\right\}$ a sequence of $H_{\mathrm{loc}}^{1}(\Omega)$. If

$$
\begin{aligned}
& \left\{\nabla_{\varepsilon} u_{\varepsilon}\right\},\left\{\varepsilon \nabla w_{\varepsilon}\right\} \text { are bounded in } L_{\mathrm{loc}}^{2}(\Omega)^{N}, \\
& u_{\varepsilon} \overrightarrow{2} u, \quad w_{\varepsilon} \overrightarrow{2} w \quad \text { in } L_{\mathrm{loc}}^{2}(\Omega \times \mathcal{Y})^{N},
\end{aligned}
$$

then $u_{\varepsilon} w_{\varepsilon} \frac{*}{2} u w$ in $C_{c}^{0}(\Omega \times \mathcal{Y})^{\prime}$.

Proof. This statement does not follow from Theorem 5.1, but may be proved via a similar (actually simpler) argument. Here $\hat{U}_{\varepsilon}, \hat{W}_{\varepsilon}, \xi_{[\varepsilon]} \hat{U}_{\varepsilon},(\mathcal{M}+\varepsilon \xi) \hat{W}_{\varepsilon}$ are uniformly bounded in $L^{2}\left(\mathbb{R}^{N} \times \mathbb{Z}^{N}\right)$, by (4.13) and (4.14).

\section{Time-dependent two-scale div-curl lemma}

In this section we extend the two-scale div-curl lemma, i.e. Theorem 5.1, to timedependent functions. Preliminarly let us fix any $T>0$, and set $\left.A_{T}:=A \times\right] 0, T[$ for any $A \subset \mathbb{R}^{N}$. We still use the notation (1.9), (1.13)-(1.15).

Theorem 6.1. Let $\left\{u_{\varepsilon}^{(1)}\right\},\left\{u_{\varepsilon}^{(2)}\right\},\left\{w_{\varepsilon}^{(1)}\right\},\left\{w_{\varepsilon}^{(2)}\right\}$ be four sequences of $L_{\mathrm{loc}}^{2}\left(\Omega_{T}\right)^{N}$ such that $\nabla \times u_{\varepsilon}^{(1)}, \nabla \times u_{\varepsilon}^{(2)} \in L_{\mathrm{loc}}^{2}\left(\Omega_{T}\right)^{N^{2}}$ and $\nabla \cdot w_{\varepsilon}^{(2)} \in L_{\mathrm{loc}}^{2}\left(\Omega_{T}\right)$ for any $\varepsilon$. If

$$
\begin{gathered}
u_{\varepsilon}:=\left(u_{\varepsilon}^{(1)}, u_{\varepsilon}^{(2)}\right) \overrightarrow{2} u:=\left(u^{(1)}, u^{(2)}\right) \\
w_{\varepsilon}:=\left(w_{\varepsilon}^{(1)}, w_{\varepsilon}^{(2)}\right) \overrightarrow{2} w:=\left(w^{(1)}, w^{(2)}\right) \\
\left\{G_{\varepsilon} \times u_{\varepsilon}\right\} \text { is bounded in } L_{\mathrm{loc}}^{2}\left(\Omega_{T}\right)^{4 N^{2}}, \\
\left\{G_{\varepsilon} \cdot w_{\varepsilon}\right\} \text { is bounded in } L_{\mathrm{loc}}^{2}\left(\Omega_{T}\right), \\
\exists r>0, \exists s>0: \text { either }\left\{u_{\varepsilon}\right\} \text { or }\left\{w_{\varepsilon}\right\} \text { is bounded in } H^{r}\left(0, T ; H^{-s}(\Omega)^{2 N}\right),
\end{gathered}
$$

then $u_{\varepsilon} \cdot w_{\varepsilon} \frac{*}{2} u \cdot w$ in $C_{c}^{0}\left(\Omega_{T} \times \mathcal{Y}\right)^{\prime}$, that is,

$$
\begin{gathered}
\iint_{\Omega_{T}}\left[u_{\varepsilon}^{(1)}(x, t) \cdot w_{\varepsilon}^{(1)}(x, t)+u_{\varepsilon}^{(2)}(x, t) \cdot w_{\varepsilon}^{(2)}(x, t)\right] \varphi(x, x / \varepsilon, t) d x d t \\
\rightarrow \iiint_{\Omega_{T} \times \mathcal{Y}}\left[u^{(1)}(x, y, t) \cdot w^{(1)}(x, y, t)+u^{(2)}(x, y, t) \cdot w^{(2)}(x, y, t)\right] \varphi(x, y, t) d x d y d t \\
\forall \varphi \in C_{c}^{0}\left(\Omega_{T} \times \mathcal{Y}\right) .
\end{gathered}
$$

Notice that, by Proposition 4.1, (6.2) and (6.3) respectively entail that

$$
G \times u \in L_{\mathrm{loc}}^{2}\left(\Omega_{T} \times \mathcal{Y}\right)^{4 N^{2}}, \quad G \cdot w \in L_{\mathrm{loc}}^{2}\left(\Omega_{T} \times \mathcal{Y}\right) .
$$


Proof. This argument mimics that of Theorem 5.1.

(i) Let us fix any $\varphi, \psi \in \mathcal{D}\left(\Omega_{T} \times \mathcal{Y}\right)$ such that $\psi=1$ on the support of $\varphi$, set

$$
\begin{aligned}
U_{\varepsilon}(x, t) & :=\left(u_{\varepsilon}^{(1)}(x, t), u_{\varepsilon}^{(2)}(x, t)\right) \varphi(x, x / \varepsilon, t) \\
W_{\varepsilon}(x, t) & :=\left(w_{\varepsilon}^{(1)}(x, t), w_{\varepsilon}^{(2)}(x, t)\right) \psi(x, x / \varepsilon, t) \quad \forall(x, y, t) \in \Omega_{T} \times \mathcal{Y}, \forall \varepsilon, \\
U(x, y, t) & :=\left(u^{(1)}(x, y, t), u^{(2)}(x, y, t)\right) \varphi(x, y, t) \quad \\
W(x, y, t) & :=\left(w^{(1)}(x, y, t), w^{(2)}(x, y, t)\right) \psi(x, y, t)
\end{aligned}
$$

and extend these functions to $\mathbb{R}^{N+1}\left(\mathbb{R}^{N+1} \times \mathcal{Y}\right.$, respectively) with vanishing value. For any $\varepsilon$ let us apply to the functions $U_{\varepsilon}(x, t)$ and $W_{\varepsilon}(x, t)$ the approximate twoscale Fourier transform (componentwise), $\mathcal{F}_{\varepsilon}$, with respect to $x(c f$. (3.4)) and the ordinary (single-scale) Fourier transform with respect to $t$. This yields two functions $\hat{U}_{\varepsilon}(\xi, \mathcal{M}, \tau)$ and $\hat{W}_{\varepsilon}(\xi, \mathcal{M}, \tau)$, that are defined for any $(\xi, \mathcal{M}, \tau) \in$ $\tilde{\mathcal{Y}}_{1 / \varepsilon} \times \mathbb{Z}^{N} \times \mathbb{R}$. For convenience of notation, henceforth we shall identify $(\xi, \mathcal{M}, \tau) \in$ $\mathbb{R}^{N} \times \mathbb{Z}^{N} \times \mathbb{R}$ with $(\xi, \tau, \mathcal{M}) \in \mathbb{R}^{N+1} \times \mathbb{Z}^{N}$.

As the functions $U_{\varepsilon}$ and $W_{\varepsilon}$ are uniformly bounded in $L^{1}\left(\mathbb{R}_{T}^{N}\right)^{2 N} \cap L^{2}\left(\mathbb{R}_{T}^{N}\right)^{2 N}$, by Proposition 3.1

$$
\hat{U}_{\varepsilon}, \hat{W}_{\varepsilon} \text { are uniformly bounded in } L^{\infty}\left(\mathbb{R}^{N+1} \times \mathbb{Z}^{N}\right)^{2 N} \cap L^{2}\left(\mathbb{R}^{N+1} \times \mathbb{Z}^{N}\right)^{2 N} \text {. }
$$

By applying the ordinary Fourier transform with respect to all variables to $U(x, y, t)$ and $W(x, y, t)$, we get two functions $\hat{U}(\xi, \mathcal{M}, \tau)$ and $\hat{W}(\xi, \mathcal{M}, \tau)$ that are defined for any $(\xi, \tau, \mathcal{M}) \in \mathbb{R}^{N+1} \times \mathbb{Z}^{N}$.

Proposition 3.2 and 3.3 have obvious extensions to time-dependent functions, which yield

$$
\begin{array}{lll}
\hat{U}_{\varepsilon} \rightarrow \hat{U}, \quad \hat{W}_{\varepsilon} \rightarrow \hat{W} & \text { pointwise in } \mathbb{R}^{N+1} \times \mathbb{Z}^{N}, \\
\hat{U}_{\varepsilon} * \hat{U}, \quad \hat{W}_{\varepsilon} * \hat{W} & \text { in } L^{\infty}\left(\mathbb{R}^{N+1} \times \mathbb{Z}^{N}\right)^{2 N} \cap L^{2}\left(\mathbb{R}^{N+1} \times \mathbb{Z}^{N}\right)^{2 N} ;
\end{array}
$$

hence, by Lebesgue's theorem, (omitting restrictions)

$$
\hat{U}_{\varepsilon} \cdot \hat{W}_{\varepsilon} \rightarrow \hat{U} \cdot \hat{W} \quad \text { in } L^{1}(C), \forall \text { bounded measurable set } C \subset \mathbb{R}^{N+1} \times \mathbb{Z}^{N} .
$$

(ii) Let us now assume that the hypothesis (6.4) holds for the sequence $\left\{u_{\varepsilon}\right\}$ (if it held for $\left\{w_{\varepsilon}\right\}$ the argument would be similar). After Corollary 4.4, by (6.2) and (6.3) we then infer that

$$
\begin{aligned}
& \hat{U}_{\varepsilon}, \quad \hat{W}_{\varepsilon},|\tau|^{r}\left|\zeta_{\varepsilon}\right|^{-s} \hat{U}_{\varepsilon}, \quad \zeta_{\varepsilon} \times \hat{U}_{\varepsilon}, \zeta_{\varepsilon} \cdot \hat{W}_{\varepsilon} \\
& \text { are uniformly bounded in } L^{2}\left(\mathbb{R}^{N+1} \times \mathbb{Z}^{N}\right)^{M} ;
\end{aligned}
$$

here either $M=1$ or $M=2 N$ or $M=4 N^{2}$, depending on the functions. (If (6.4) held for $\left\{w_{\varepsilon}\right\}$, we should replace $|\tau|^{r}\left|\zeta_{\varepsilon}\right|^{-s} \hat{U}_{\varepsilon}$ by $|\tau|^{r}\left|\zeta_{\varepsilon}\right|^{-s} \hat{W}_{\varepsilon}$ in this formula, but 
the remainder would be unchanged.) By applying the identity (5.12) as above and using (4.3), we infer that

$$
\left\|\left(1+|\tau|^{r}\left|\zeta_{\varepsilon}\right|^{-s}+\left|\zeta_{\varepsilon}\right|\right) \hat{U}_{\varepsilon} \cdot \hat{W}_{\varepsilon}\right\|_{L^{1}\left(\tilde{\mathcal{Y}}_{1 / \varepsilon} \times \mathbb{R} \times \mathbb{Z}^{N}\right)} \leq \text { Constant. }
$$

Let us now set

$$
\beta:=\frac{r}{s+1}, \quad \text { whence } \quad \frac{\beta}{r}+\frac{s \beta}{r}=1 .
$$

The Young inequality yields

$$
|\tau|^{\beta}=\left(|\tau|^{r}\left|\zeta_{\varepsilon}\right|^{-s}\right)^{\beta / r}\left|\zeta_{\varepsilon}\right|^{s \beta / r} \leq \frac{\beta}{r}|\tau|^{r}\left|\zeta_{\varepsilon}\right|^{-s}+\frac{s \beta}{r}\left|\zeta_{\varepsilon}\right|,
$$

whence

$$
\begin{aligned}
& \left\||\tau|^{\beta} \hat{U}_{\varepsilon} \cdot \hat{W}_{\varepsilon}\right\|_{L^{1}\left(\tilde{\mathcal{Y}}_{1 / \varepsilon} \times \mathbb{R} \times \mathbb{Z}^{N}\right)} \\
& \leq \frac{\beta}{r}\left\||\tau|^{r}\left|\zeta_{\varepsilon}\right|^{-s} \hat{U}_{\varepsilon} \cdot \hat{W}_{\varepsilon}\right\|_{L^{1}\left(\tilde{\mathcal{Y}}_{1 / \varepsilon} \times \mathbb{R} \times \mathbb{Z}^{N}\right)}+\frac{s \beta}{r}\left\|\left|\zeta_{\varepsilon}\right| \hat{U}_{\varepsilon} \cdot \hat{W}_{\varepsilon}\right\|_{L^{1}\left(\tilde{\mathcal{Y}}_{1 / \varepsilon} \times \mathbb{Z}^{N}\right)} \\
& \leq \text { Constant independent of } \varepsilon
\end{aligned}
$$

By (6.8) we then get

$$
\hat{U}_{\varepsilon} \cdot \hat{W}_{\varepsilon} \rightarrow \hat{U} \cdot \hat{W} \quad \text { in } L^{1}\left(\mathbb{R}^{N+1} \times \mathbb{Z}^{N}\right) .
$$

The reminder of the argument mimics part (iii) of Theorem 5.1, and is here omitted.

Proposition 5.2 may also be extended to the time dependent setting, but we refrain from developing this issue, due to the awkwardness of the hypotheses. We rather state a straightforward extension of Proposition 5.3.

Proposition 6.2. Let $\left\{u_{\varepsilon}\right\}$ be a sequence of $L_{\mathrm{loc}}^{2}\left(\Omega_{T}\right)$ and $\left\{w_{\varepsilon}\right\}$ a sequence of $H_{\mathrm{loc}}^{1}\left(\Omega_{T}\right)$. If

$$
\left\{\nabla_{\varepsilon} u_{\varepsilon}\right\},\left\{\varepsilon \nabla w_{\varepsilon}\right\} \text { are bounded in } L_{\text {loc }}^{2}\left(\Omega_{T}\right)^{N},
$$

$\exists r>0, \exists s>0$ : either $\left\{u_{\varepsilon}\right\}$ or $\left\{w_{\varepsilon}\right\}$ is bounded in $H^{r}\left(0, T ; H^{-s}(\Omega)\right)$,

$$
u_{\varepsilon} \overrightarrow{2} u, \quad w_{\varepsilon} \overrightarrow{2} w \quad \text { in } L_{\mathrm{loc}}^{2}\left(\Omega_{T} \times \mathcal{Y}\right),
$$

then $u_{\varepsilon} w_{\varepsilon} \overrightarrow{2} u w$ in $C_{c}^{0}\left(\Omega_{T} \times \mathcal{Y}\right)^{\prime}$.

\section{Two-scale Maxwell system}

This section is aimed to introduce a two-scale formulation of the Maxwell equations, providing a setting in which the two-scale div-curl Theorems 5.1 and 6.1 may be applied. 


\section{Asymptotic expansions}

Let us first consider the formal asymptotic expansion of a field of $x \in \mathbb{R}^{3}$ with respect to a parameter $\varepsilon>0$ :

$$
u_{\varepsilon}(x)=\sum_{n \geq 0} \varepsilon^{n} u_{n}(x, x / \varepsilon)=u_{0}(x, x / \varepsilon)+\varepsilon u_{1}(x, x / \varepsilon)+O\left(\varepsilon^{2}\right) \quad x \in \mathbb{R}^{3},
$$

the functions $u_{n}$ being $Y$-periodic with respect to the second argument. Here we may recognize the dependence on both a coarse and a fine length-scale, that are respectively represented by the variables $x$ and $y:=x / \varepsilon$. Denoting by $\nabla$ the total gradient with respect to $x$ and by $\nabla_{x}\left(\nabla_{y}\right.$, respectively) the partial gradient with respect to the first (second, respectively) variable, formally (7.1) yields

$$
\begin{aligned}
\nabla u_{\varepsilon}(x)= & \sum_{n \geq 0}\left[\varepsilon^{n} \nabla_{x} u_{n}(x, x / \varepsilon)+\varepsilon^{n-1} \nabla_{y} u_{n}(x, x / \varepsilon)\right] \\
= & \nabla_{x} u_{0}(x, x / \varepsilon)+\varepsilon^{-1} \nabla_{y} u_{0}(x, x / \varepsilon)+\varepsilon \nabla_{x} u_{1}(x, x / \varepsilon) \\
& +\nabla_{y} u_{1}(x, x / \varepsilon)+O(\varepsilon)
\end{aligned}
$$

for $x \in \mathbb{R}^{3}$. As this equality must be fulfilled for any small $\varepsilon$, one is induced to assume that $\nabla_{y} u_{0} \equiv 0$. This construction provides the heuristic basis of the classical theory of homogenization, and was extensively investigated by applied scientists and mathematicians, see $e . g$. [5, 6, 24].

A different setting arises by allowing $u_{\varepsilon}$ to have $O(1)$ variations at the $\varepsilon$ lengthscale; e.g., $u_{\varepsilon}(x)=x \sin (2 \pi|x| / \varepsilon)$. Rather than considering the total gradient, in this case it seems natural to deal with the two-scale differential operators $\nabla_{x}$ and $\nabla_{y}$, that may respectively be approximated by $\nabla_{\varepsilon}$ and $\varepsilon \nabla, c f$. Proposition 1.2 and [31]. Here we outline a two-scale formulation of the Maxwell system of electromagnetism, couple it with a nonlinear relation between the fields $B$ and $H$, and briefly illustrate the procedure of approximation and passage to the limit.

\section{Reformulation of the single-scale Maxwell system}

Consistently with the convention that is usually assumed in physics, in this section we shall regard vector products and curls of vector fields of $\mathbb{R}^{3}$ as elements of $\mathbb{R}^{3}$, rather than $3 \times 3$-tensors. We shall then transform these vectors to tensors, via the Levi-Civita-tensor.

Let us first review the classical (single-scale) Maxwell system. In order to mitigate the intrinsic complexity of the two-scale extension that will follow, we neglect the displacement current $\partial D / \partial t$ from the beginning, and afterwards will also assume the (linear) Ohm law. It is known that this approximation is physically acceptable in metals, $c f$. e.g. [14]. In the rationalized MKSA unit system, the Faraday 
and Ampère equations read

$$
\left\{\begin{array}{l}
\frac{\partial B}{\partial t}+\nabla \times E=0 \\
J=\nabla \times H+r
\end{array} \quad \text { in } \mathbb{R}^{3} \times\right] 0, T[
$$

here $r$ is a prescribed source-term that represents a divergence-free electric-current density due to some applied electromagnetic force. Hence $\nabla \cdot \partial B / \partial t=0$ and this entails the Gauss law

$$
\left.\nabla \cdot B=0 \quad \text { in } \mathbb{R}^{3} \times\right] 0, T[,
$$

provided that (7.4) itself is fulfilled at some instant. We shall now rewrite this system in a form that is more prone to the two-scale extension. Let us first define the Levi-Civita antisymmetric unit pseudo-tensor:

$$
\begin{array}{ll}
\epsilon_{i j k}=1 & \text { if }(i, j, k)=(1,2,3) \text {, or }(i, j, k)=(2,3,1), \text { or }(i, j, k)=(3,1,2), \\
\epsilon_{i j k}=-1 & \text { if }(i, j, k)=(3,2,1) \text {, or }(i, j, k)=(1,3,2), \text { or }(i, j, k)=(2,1,3), \\
\epsilon_{i j k}=0 & \text { otherwise. }
\end{array}
$$

Let us also assume the convention of the sum over repeated indices, and define the antisymmetric second order tensors

$$
R_{i j}=\epsilon_{i j k} E_{k}, \quad S_{i j}=\epsilon_{i j k} J_{k}, \quad g_{i j}=\epsilon_{i j k} r_{k} \quad(j, k=1,2,3),
$$

so that

$$
(\nabla \times E)_{i}=\nabla_{j} R_{i j}, \quad J_{k}=\epsilon_{i j k} S_{i j}, \quad r_{k}=\epsilon_{i j k} g_{i j} \quad(i, j, k=1,2,3) .
$$

The system (7.3) then also reads

$$
\left\{\begin{array}{l}
\frac{\partial B_{i}}{\partial t}+\nabla_{j} R_{i j}=0 \\
S_{i j}=\nabla_{i} H_{j}-\nabla_{j} H_{i}+g_{i j}
\end{array} \quad \text { in } \mathbb{R}^{3} \times\right] 0, T[(i=1,2,3) .
$$

Let us now prescribe the Ohm law $E=\rho J$, namely $R=\rho S$, the resistance $\rho=\rho(x)$ being a positive scalar field (more generally a symmetric tensor for an anisotropic material). Setting $f_{i}:=-\nabla_{j}\left(\rho g_{i j}\right)$, this system is thus reduced to a single (vector) equation:

$$
\left.\frac{\partial B_{i}}{\partial t}+\nabla_{j}\left[\rho\left(\nabla_{i} H_{j}-\nabla_{j} H_{i}\right)\right]=f_{i} \quad \text { in } \mathbb{R}^{3} \times\right] 0, T[(i=1,2,3) .
$$

This is equivalent to $\partial B / \partial t+\nabla \times[\rho(\nabla \times H)]=f$, but is written in a form that we can easily extend to the two-scale setting. 


\section{Two-scale Maxwell system}

We shall deal with fields that depend on both the coarse- and fine-scale variables $x, y$, and that are $Y$-periodic with respect to to $y$; we may thus identify any of them with its restriction to $\mathbb{R}^{3} \times \mathcal{Y}$. Having doubled the independent variables, we also need to double the components of the electro-magnetic fields. For instance, in the magnetostatic setting (i.e., in the absence of Ampèrian and displacement electric currents) it is natural to assume that $H=H(x, y)$ is the gradient of a scalar $Y$ periodic potential of the form $\varphi(x, y)$, that is, $H^{\prime}=\left(\nabla_{x} \varphi, \nabla_{y} \varphi\right)$ in $\mathbb{R}^{3}$. We shall label 6-component vectors by a prime. Defining the average and fluctuating parts $\hat{u}$ and $\tilde{u}$ of any field $u=u(x, y)$,

$$
\hat{u}(x):=\int_{Y} u(x, y) d y, \quad \tilde{u}(x, y):=u(x, y)-\hat{u}(x),
$$

one has $\varphi(x, y)=\hat{\varphi}(x)+\tilde{\varphi}(x, y)$, and

$$
H^{\prime}(x, y)=\hat{H}^{\prime}(x)+\tilde{H}^{\prime}(x, y)=\left(\nabla_{x} \hat{\varphi}(x), 0\right)+\left(\nabla_{x} \tilde{\varphi}(x, y), \nabla_{y} \tilde{\varphi}(x, y)\right) .
$$

Thus $\tilde{H}^{\prime}$ depends on $x$ whenever the fine-scale structure is not uniform on the coarse-scale.

We shall represent these $\mathbb{R}^{6}$-valued vectors as the tensor product of two $\mathbb{R}^{3}$ valued vectors, that we respectively label by the indices $a$ and $b ; e . g$.

$$
B^{\prime}:=\left(B_{a}, B_{b}\right)=\left(B_{1}, \ldots, B_{6}\right), \quad H^{\prime}:=\left(H_{a}, H_{b}\right)=\left(H_{1}, \ldots, H_{6}\right) .
$$

We also define the $\left(\mathbb{R}^{6}\right)^{2}$-valued tensor fields

$$
R^{\prime}:=\left(\begin{array}{ll}
R_{a a} & R_{a b} \\
R_{b a} & R_{b b}
\end{array}\right), \quad S^{\prime}:=\left(\begin{array}{cc}
S_{a a} & S_{a b} \\
S_{b a} & S_{b b}
\end{array}\right), \quad g^{\prime}:=\left(\begin{array}{ll}
g_{a a} & g_{a b} \\
g_{b a} & g_{b b}
\end{array}\right),
$$

that we assume to be antisymmetric:

$$
R_{i j}^{\prime}=-R_{j i}^{\prime}, \quad S_{i j}^{\prime}=-S_{j i}^{\prime}, \quad g_{i j}^{\prime}=-g_{j i}^{\prime} \quad(i, j=1, \ldots, 6) .
$$

We shall label $6^{2}$-component tensors by a prime, too. We also replace the gradient $\nabla$ by the 6-component differential operator $\nabla^{\prime}:=\left(\nabla_{x}, \nabla_{y}\right)=\left(\nabla_{1}, \ldots, \nabla_{6}\right)$.

Next we generalize the single-scale system (7.6) to the two-scale framework by setting

$$
\left\{\begin{array}{l}
\frac{\partial B_{i}^{\prime}}{\partial t}+\nabla_{j}^{\prime} R_{i j}^{\prime}=0 \\
S_{i j}^{\prime}=\nabla_{i}^{\prime} H_{j}^{\prime}-\nabla_{j}^{\prime} H_{i}^{\prime}+g_{i j}^{\prime}
\end{array} \quad \text { in } \mathbb{R}^{3} \times \mathcal{Y} \times\right] 0, T[(i=1, \ldots, 6) .
$$

Because of the antisymmetry of $R^{\prime}$, the first of these equations entails $\nabla_{i} \partial B_{i}^{\prime} / \partial t=$ 0 ; this yields the two-scale Gauss law

$$
\left.\nabla^{\prime} \cdot B^{\prime}=0 \quad \text { in } \mathbb{R}^{3} \times \mathcal{Y} \times\right] 0, T[
$$


provided that (7.12) itself is fulfilled at some instant. (The necessity of deriving this law is one of the main difficulties that underlie this two-scale approach, and is here solved via the introduction of 6-component fields.) By assuming the two-scale Ohm law

$$
R^{\prime}=\rho S^{\prime} \quad \text { with } \rho=\rho(x, y)>0
$$

and setting $f_{i}^{\prime}:=-\nabla_{j} g_{i j}^{\prime}$, the first-order system (7.11) may be written as a secondorder 6-component equation:

$$
\left.\frac{\partial B_{i}^{\prime}}{\partial t}+\nabla_{j}\left[\rho\left(\nabla_{i} H_{j}^{\prime}-\nabla_{j} H_{i}^{\prime}\right)\right]=f_{i}^{\prime} \quad \text { in } \mathbb{R}^{3} \times \mathcal{Y} \times\right] 0, T[(i=1, \ldots, 6) .
$$

For instance if $\rho$ is constant ( $\rho=1$, say) then this equation is reduced to

$$
\left.\frac{\partial B_{i}^{\prime}}{\partial t}+\nabla_{i} \nabla_{j} H_{j}^{\prime}-\nabla_{j} \nabla_{j} H_{i}^{\prime}=f_{i}^{\prime} \quad \text { in } \mathbb{R}^{3} \times \mathcal{Y} \times\right] 0, T[(i=1, \ldots, 6),
$$

that also reads

$$
\left\{\begin{array}{l}
\frac{\partial B_{a}}{\partial t}+\nabla_{x}\left(\nabla_{x} \cdot H_{a}+\nabla_{y} \cdot H_{b}\right)-\Delta_{x} H_{a}-\Delta_{y} H_{a}=f_{a} \\
\frac{\partial B_{b}}{\partial t}+\nabla_{y}\left(\nabla_{x} \cdot H_{a}+\nabla_{y} \cdot H_{b}\right)-\Delta_{x} H_{b}-\Delta_{y} H_{b}=f_{b}
\end{array} \quad \text { in } \mathbb{R}^{3} \times \mathcal{Y} \times\right] 0, T[,
$$

that is, as $-\Delta=\nabla \times \nabla \times-\nabla \nabla \cdot\left(\right.$ i.e., $-\Delta=$ curl $^{2}-\operatorname{grad}$ div $)$,

$$
\left\{\begin{array}{l}
\frac{\partial B_{a}}{\partial t}+\nabla_{x} \times \nabla_{x} \times H_{a}+\nabla_{x}\left(\nabla_{y} \cdot H_{b}\right)-\Delta_{y} H_{a}=f_{a} \\
\frac{\partial B_{b}}{\partial t}+\nabla_{y} \times \nabla_{y} \times H_{b}+\nabla_{y}\left(\nabla_{x} \cdot H_{a}\right)-\Delta_{x} H_{b}=f_{b}
\end{array} \quad \text { in } \mathbb{R}^{3} \times \mathcal{Y} \times\right] 0, T[.
$$

The average and the fluctuating part of this system respectively read

$$
\begin{gathered}
\left\{\begin{array}{l}
\frac{\partial \hat{B}_{a}}{\partial t}+\nabla_{x} \times \nabla_{x} \times \hat{H}_{a}=\hat{f}_{a} \\
\frac{\partial \hat{B}_{b}}{\partial t}-\Delta_{x} \hat{H}_{b}=\hat{f}_{b}
\end{array} \quad \text { in } \mathbb{R}^{3} \times\right] 0, T[, \\
\left\{\begin{array}{l}
\frac{\partial \tilde{B}_{a}}{\partial t}+\nabla_{x} \times \nabla_{x} \times \tilde{H}_{a}+\nabla_{x}\left(\nabla_{y} \cdot \tilde{H}_{b}\right)-\Delta_{y} \tilde{H}_{a}=\tilde{f}_{a} \\
\frac{\partial \tilde{B}_{b}}{\partial t}+\nabla_{y} \times \nabla_{y} \times \tilde{H}_{b}+\nabla_{y}\left(\nabla_{x} \cdot \tilde{H}_{a}\right)-\Delta_{x} \tilde{H}_{b}=\tilde{f}_{b}
\end{array}\right. \\
\text { in } \left.\mathbb{R}^{3} \times \mathcal{Y} \times\right] 0, T[.
\end{gathered}
$$

One may thus replace (7.16) by the two latter systems, that are mutually uncoupled. Notice that the equation $(7.17)_{2}$ may be dropped, for the fields $\hat{B}_{b}, \hat{H}_{b}$ do not occur elsewhere. Moreover the system (7.18) yields

$$
\left.\nabla_{x} \cdot \frac{\partial \tilde{B}_{a}}{\partial t}+\nabla_{y} \cdot \frac{\partial \tilde{B}_{b}}{\partial t}=\nabla_{x} \cdot \tilde{f}_{a}+\nabla_{y} \cdot \tilde{f}_{b} \quad \text { in } \mathbb{R}^{3} \times \mathcal{Y} \times\right] 0, T[.
$$


If $\nabla_{x} \cdot \tilde{f}_{a}+\nabla_{y} \cdot \tilde{f}_{b}=0$ we then retrieve the two-scale Gauss law (7.12),

$$
\left.\nabla_{x} \cdot B_{a}+\nabla_{y} \cdot B_{b}=\nabla_{x} \cdot \tilde{B}_{a}+\nabla_{y} \cdot \tilde{B}_{b}=0 \quad \text { in } \mathbb{R}^{3} \times \mathcal{Y} \times\right] 0, T[,
$$

provided that this is fulfilled at some instant. We are left with the selection of a constitutive relation between $B^{\prime}$ and $H^{\prime}$. For instance one might prescribe a condition of the form

$$
\left.B^{\prime} \in \partial \varphi\left(H^{\prime}, x, y\right) \quad \text { in } \mathbb{R}^{3} \times \mathcal{Y} \times\right] 0, T[,
$$

where $\varphi: \mathbb{R}^{6} \times \mathbb{R}^{3} \times \mathcal{Y}$ is convex and lower semicontinuous with respect to the first argument, and $\partial \varphi$ is the subdifferential with respect to that argument, $c f . e . g$. $[12,13]$.

Let us now multiply the equation (7.15) by $H_{i}^{\prime}$, sum for $i=1, \ldots, 6$, and integrate in space and time. Denoting by $\varphi^{*}(\cdot, x, y)$ the convex conjugate function of $\varphi(\cdot, x, y)$, we get the two-scale form of the energy integral:

$$
\begin{aligned}
& \iint_{\mathbb{R}^{3} \times \mathcal{Y}}\left[\varphi^{*}\left(B^{\prime}(x, y, t), x, y\right)-\varphi^{*}\left(B^{\prime}(x, y, 0), x, y\right)\right] d x d y \\
& \quad+\sum_{i, j=1}^{6} \iiint_{\left.\mathbb{R}^{3} \times \mathcal{Y} \times\right] 0, t[}\left|\nabla_{j} H_{i}^{\prime}-\nabla_{i} H_{j}^{\prime}\right|^{2} d x d y d \tau \\
& \left.=\sum_{i=1}^{6} \iiint_{\left.\mathbb{R}^{3} \times \mathcal{Y} \times\right] 0, t[} f_{i}^{\prime} \cdot H_{i}^{\prime} d x d y d \tau \quad \text { for a.e. } t \in\right] 0, T[.
\end{aligned}
$$

This accounts for the energy embedded in both coarse- and fine-scale oscillations.

Let us now point out a particular case. If $B_{a}$ only depends on $x$ and $B_{b}$ only on $y$, then (7.17) and (7.18) are reduced to two uncoupled equations:

$$
\left\{\begin{array}{l}
\frac{\partial \hat{B}_{a}}{\partial t}+\nabla_{x} \times \nabla_{x} \times \hat{H}_{a}=\hat{f}_{a} \\
\frac{\partial \tilde{B}_{b}}{\partial t}+\nabla_{y} \times \nabla_{y} \times \tilde{H}_{b}=\tilde{f}_{b}
\end{array} \quad \text { in } \mathbb{R}^{3} \times \mathcal{Y} \times\right] 0, T[.
$$

This example may cast some light upon the role of the pairs $\left(\hat{B}_{a}, \hat{H}_{a}\right)$ and $\left(\tilde{B}_{b}, \tilde{H}_{b}\right)$, although it rests on the rather strong assumption that the latter pair be independ of $x$. In general instead different macroscopic points will be in different mesoscopic states and will have different deviations from the average field, but for the case in which the constitutive function $\varphi$ is linear and independent of $x$. Indeed the role of the coarse- and fine-scale variables $x, y$ need not be symmetric.

\section{Application of the two-scale div-curl lemma}

Next we couple the equation (7.15) with the constitutive relation (7.19) and with the initial condition

$$
B^{\prime}(x, y, 0)=B^{0^{\prime}}(x, y) \quad \text { in } \mathbb{R}^{3} \times \mathcal{Y},
$$


for a prescribe field $B^{0^{\prime}}: \mathbb{R}^{3} \times \mathcal{Y} \rightarrow \mathbb{R}^{6}$. We shall briefly outline how existence of a weak solution can be proved for this system via an argument based on the singlescale approximation and Theorem 6.1, namely the two-scale div-curl lemma. Setting

For any $\varepsilon>0$ we approximate $\nabla^{\prime}$ by $\nabla_{\varepsilon}^{\prime}:=\left(\nabla_{\varepsilon}, \varepsilon \nabla\right)=\left(\nabla_{\varepsilon 1}^{\prime}, \ldots, \nabla_{\varepsilon 6}^{\prime}\right)$.

$$
B_{\varepsilon}^{\prime}=\left(B_{\varepsilon a}, B_{\varepsilon b}\right)=\left(B_{\varepsilon 1}, \ldots, B_{\varepsilon 6}\right), \quad H_{\varepsilon}^{\prime}=\left(H_{\varepsilon a}, H_{\varepsilon b}\right)=\left(H_{\varepsilon 1}, \ldots, H_{\varepsilon 6}\right),
$$

a natural approximation of (7.14) reads

$$
\left\{\begin{array}{l}
\frac{\partial B_{\varepsilon i}^{\prime}}{\partial t}+\nabla_{\varepsilon j}^{\prime}\left(\nabla_{\varepsilon i}^{\prime} H_{\varepsilon j}^{\prime}-\nabla_{\varepsilon j}^{\prime} H_{\varepsilon i}^{\prime}\right)=f_{i} \\
B_{\varepsilon}^{\prime} \in \partial \varphi\left(H_{\varepsilon}^{\prime}, x, x / \varepsilon\right)
\end{array} \quad \text { in } \mathbb{R}^{3} \times\right] 0, T[(i=1, \ldots, 6),
$$

that is,

$$
\left\{\begin{array}{l}
\frac{\partial B_{\varepsilon a}}{\partial t}+\nabla_{\varepsilon} \times \nabla_{\varepsilon} \times H_{\varepsilon a}+\nabla_{\varepsilon}\left(\varepsilon \nabla \cdot H_{\varepsilon b}\right)-\varepsilon^{2} \nabla \cdot \nabla H_{\varepsilon a}=f_{a} \\
\left.\frac{\partial B_{\varepsilon b}}{\partial t}+\varepsilon^{2} \nabla \times \nabla \times H_{\varepsilon b}+\varepsilon \nabla\left(\nabla_{\varepsilon} \cdot H_{\varepsilon a}\right)-\nabla_{\varepsilon} \cdot \nabla_{\varepsilon} H_{\varepsilon b}=f_{b} \quad \text { in } \mathbb{R}^{3} \times\right] 0, T[. \\
\left(B_{\varepsilon a}, B_{\varepsilon b}\right) \in \partial \varphi\left(H_{\varepsilon a}, H_{\varepsilon b}, x, x / \varepsilon\right)
\end{array}\right.
$$

We append the initial conditions

$$
B_{\varepsilon a}(x, 0)=B_{\varepsilon a}^{0}(x), \quad B_{\varepsilon b}(x, 0)=B_{\varepsilon b}^{0}(x) \quad \text { for almost all } x \in \mathbb{R}^{3},
$$

and assume that

$$
\begin{aligned}
& f_{a}, f_{b} \in L^{2}\left(\mathbb{R}^{3} \times\right] 0, T[)^{3}, \\
& B_{\varepsilon a} \rightarrow B_{a}^{0}, \quad B_{\varepsilon b} \rightarrow B_{b}^{0} \quad \text { in } L^{2}\left(\mathbb{R}^{3} \times \mathcal{Y} \times\right] 0, T[)^{3}, \\
& \nabla_{\varepsilon} \cdot B_{a}^{0}+\varepsilon \nabla \cdot B_{b}^{0} \rightarrow \nabla_{x} \cdot B_{a}^{0}+\nabla_{y} \cdot B_{b}^{0}=0 \quad \text { in } L^{2}\left(\mathbb{R}^{3} \times \mathcal{Y} \times\right] 0, T[),
\end{aligned}
$$

the potential $\varphi(\cdot, x, y)$ has quadratic growth at infinity, uniformly with respect to $x, y$.

The first two equations of (7.24) also read

$$
\left.\frac{\partial}{\partial t}\left(B_{\varepsilon a}, B_{\varepsilon b}\right)+\mathcal{A}_{\varepsilon}\left(H_{\varepsilon a}, H_{\varepsilon b}\right)=\left(f_{a}, f_{b}\right) \quad \text { in } \mathbb{R}^{3} \times\right] 0, T[,
$$

where $\mathcal{A}_{\varepsilon}$ is a degenerate elliptic operator. By the classical theory of nonlinear parabolic equations (cf. e.g. $[8,17])$, for any $\varepsilon>0$ the problem $(7.24),(7.25)$ has a weak solution. Moreover, by an approximated version of the energy integral (7.20),

$$
\begin{aligned}
& B_{\varepsilon a}, B_{\varepsilon b} \in L^{2}\left(\mathbb{R}^{3} \times\right] 0, T[)^{3} \cap H^{1}\left(0, T ; H^{-1}\left(\mathbb{R}^{3}\right)^{3}\right), \\
& H_{\varepsilon a}, H_{\varepsilon b} \in L^{2}\left(0, T ; L_{\mathrm{rot}}^{2}\left(\mathbb{R}^{3}\right)^{3}\right) .
\end{aligned}
$$


Moreover, multiplying (7.24) 1 and (7.24) 2 respectively by $H_{\varepsilon a}$ and $H_{\varepsilon b}$ one derives the energy estimate, whence one infers that

$$
\begin{gathered}
B_{\varepsilon a}, B_{\varepsilon b}, H_{\varepsilon a}, H_{\varepsilon b} \text { are uniformly bounded in } L^{2}\left(\mathbb{R}^{3} \times\right] 0, T[)^{3}, \\
\nabla_{\varepsilon} \times H_{\varepsilon a}, \varepsilon \nabla \times H_{\varepsilon a}, \nabla_{\varepsilon} \times H_{\varepsilon b}, \varepsilon \nabla \times H_{\varepsilon b} \text { are uniformly bounded in } \\
L^{2}\left(\mathbb{R}^{3} \times\right] 0, T[)^{3},
\end{gathered}
$$

$B_{\varepsilon a}, B_{\varepsilon b}$ are uniformly bounded in $H^{1}\left(0, T ; H^{-1}\left(\mathbb{R}^{3}\right)^{3}\right)$.

Therefore there exist $B_{a}, B_{b}, H_{a}, H_{b}$ such that, letting $\varepsilon$ vanish along a suitable sequence,

$B_{\varepsilon a} \underset{2}{\rightarrow} B_{a}, \quad B_{\varepsilon b} \underset{2}{\rightarrow} B_{b}, \quad H_{\varepsilon a} \underset{2}{\rightarrow} H_{a}, \quad H_{\varepsilon b} \underset{2}{\rightarrow} H_{b}$ in $L^{2}\left(\mathbb{R}^{3} \times \mathcal{Y} \times\right] 0, T[)^{3}$,

and by Proposition 1.2

$$
\begin{aligned}
& \nabla_{\varepsilon} \times H_{\varepsilon a} \underset{2}{\rightarrow} \nabla_{x} \times H_{a}, \quad \nabla_{\varepsilon} \times H_{\varepsilon b} \underset{2}{\rightarrow} \nabla_{x} \times H_{b} \\
& \varepsilon \nabla \times H_{\varepsilon a} \underset{2}{\rightarrow} \nabla_{y} \times H_{a}, \quad \varepsilon \nabla \times H_{\varepsilon b} \underset{2}{\rightarrow} \nabla_{y} \times H_{b}
\end{aligned} \text { in } L^{2}\left(\mathbb{R}^{3} \times \mathcal{Y} \times\right] 0, T[)^{3} .
$$

By passing to the limit in $(7.24)_{1}$ and $(7.24)_{2}$ we then get

$$
\begin{cases}\frac{\partial B_{a}}{\partial t}+\nabla_{x} \times \nabla_{x} \times H_{a}+\nabla_{x}\left(\nabla_{y} \cdot H_{b}\right)-\Delta_{y} H_{a}=f_{a} & \\ \frac{\partial B_{b}}{\partial t}+\nabla_{y} \times \nabla_{y} \times H_{b}+\nabla_{y}\left(\nabla_{x} \cdot H_{a}\right)-\Delta_{x} H_{b}=f_{b} & \text { in } \left.\mathbb{R}^{3} \times \mathcal{Y} \times\right] 0, T[.\end{cases}
$$

We already saw that this system is tantamount to the system (7.15).

We are left with the passage to the limit in the nonlinear relation (7.24) 3 . First we notice that the equation $(7.23)_{1}$ also yields $\nabla_{\varepsilon}^{\prime} \cdot \partial B_{\varepsilon} / \partial t=0$, whence by $(7.24)_{3}$

$$
\nabla_{\varepsilon}^{\prime} \cdot B_{\varepsilon}^{\prime}(x, t)=\nabla_{\varepsilon}^{\prime} \cdot B_{\varepsilon}^{\prime}(x, 0)=\nabla_{\varepsilon}^{\prime} \cdot B_{\varepsilon}^{0^{\prime}}(x) \underset{2}{\rightarrow} 0 \text { in } L^{2}\left(\mathbb{R}^{3} \times \mathcal{Y} \times\right] 0, T[) .
$$

By applying Theorem 6.1 with $N=3, u_{\varepsilon}=H_{\varepsilon}^{\prime}$ and $w_{\varepsilon}=B_{\varepsilon}^{\prime}$, we then get the key property

$$
\begin{gathered}
\iint_{\left.\mathbb{R}^{3} \times\right] 0, T[} B_{\varepsilon}^{\prime}(x, t) \cdot H_{\varepsilon}^{\prime}(x, t) \varphi(x, x / \varepsilon, t) d x d t \\
\rightarrow \iiint_{\left.\mathbb{R}^{3} \times \mathcal{Y} \times\right] 0, T[} B^{\prime}(x, y, t) \cdot H^{\prime}(x, y, t) \varphi(x, y, t) d x d y d t \\
\forall \varphi \in C_{c}^{0}\left(\mathbb{R}^{3} \times \mathcal{Y} \times\right] 0, T[) .
\end{gathered}
$$

By the two-scale extension of a standard procedure (cf. e.g. [34, Theorem 3.1]), this allows us to pass to the limit in the nonlinear relation $(7.23)_{2}$, and thus to prove (7.19).

In conclusion, we have outlined how under the hypotheses (7.26) one can prove existence of a weak solution of the problem (7.15), (7.19), (7.22) via singlescale approximation. After time integration, the uniqueness of the solution is easily proved whenever $\varphi$ is strictly convex. 


\section{Discussion}

The two-scale formulation of the Maxwell equations may be of interest in cases in which the relevant fields exhibit large fine-scale oscillations, for it accounts for the energy embedded in both coarse- and fine-scale oscillations.

We applied the two-scale div-curl lemma to derive (7.33). However in this setting this convergence can also be retrieved via a standard lower semicontinuity argument, that here we just outline. Integrating the equation (7.15) in time and multiplying it by $H^{\prime}$, we have

$$
\begin{gathered}
\iiint_{\left.\mathbb{R}^{3} \times \mathcal{Y} \times\right] 0, t[}\left(B_{i}^{\prime} \cdot H_{i}^{\prime}-\int_{0}^{t} f_{i}^{\prime} d \tau \cdot H_{i}^{\prime}\right) d x d y d \tau \\
+\frac{1}{2} \iint_{\mathbb{R}^{3} \times \mathcal{Y}}\left|\nabla^{\prime} \times \int_{0}^{t} H^{\prime} d \tau\right|^{2} d x d y=0
\end{gathered}
$$

for any $t \in] 0, T]$. The $f^{\prime}$-term is weakly continuous and the second integral is weakly lower semicontinuous; this allows one to pass to the limit in the maximal monotone inclusion $(7.23)_{2}$.

Above we illustrated the application of the two-scale div-curl lemma to a model problem. Actually the limit procedure that we outlined is more significant in cases in which other techniques might fail, e.g. in presence either of a nonsymmetric conductivity or of a source term that depends nonlinearly either on $B^{\prime}$ or on $H^{\prime}$.

Finally we briefly discuss two attempts to preserve the 3-component structure for the two-scale Maxwell system; this author regards both alternatives as unsatisfactory.

(i) One might simply replace the single-scale operator $\nabla$ by the two-scale one $a \nabla_{x}+b \nabla_{y}$, where $a, b$ are two positive constants that for the sake of simplicity we may assume to equal 1. In place of (7.7) this would yield (for $\rho=1$, say)

$$
\left.\frac{\partial B}{\partial t}+\left(\nabla_{x}+\nabla_{y}\right) \times\left[\left(\nabla_{x}+\nabla_{y}\right) \times H\right]=f \quad \text { in } \mathbb{R}^{3} \times \mathcal{Y} \times\right] 0, T[,
$$

whence one would retrieve the two-scale Gauss law in the form

$$
\left.\left(\nabla_{x}+\nabla_{y}\right) \cdot B=0 \quad \text { in } \mathbb{R}^{3} \times \mathcal{Y} \times\right] 0, T[.
$$

In this case multiplying the equation (7.35) by $H$ one gets

$$
\iiint_{\left.\mathbb{R}^{3} \times \mathcal{Y} \times\right] 0, T[}\left(\frac{\partial B}{\partial t} \cdot H+\left|\left(\nabla_{x}+\nabla_{y}\right) \times H\right|^{2}-f \cdot H\right) d x d y d t=0
$$

if a cyclically monotone relation is assumed between $H$ and $B$, the first addendum may be integrated in time. This yields the energy integral, whence $\left(\nabla_{x}+\nabla_{y}\right) \times H \in$ $L^{2}\left(\mathbb{R}^{3} \times \mathcal{Y} \times\right] 0, T[)^{3}$; but this does not entail the separate square-integrability of $\nabla_{x} \times H$ and $\nabla_{y} \times H$, for

$$
\left|\left(\nabla_{x}+\nabla_{y}\right) \times H\right|^{2}=\left|\nabla_{x} \times H\right|^{2}+\left|\nabla_{y} \times H\right|^{2}+\nabla_{x} \times \nabla_{y} \times H+\nabla_{y} \times \nabla_{x} \times H,
$$


and the sum of the two latter addenda need not vanish. One can nevertheless approximate (7.35) by a single-scale equation, and also pass to the limit in the nonlinear relation via a semicontinuity argument, without the need of using compensated compactness.

(ii) In alternative one might replace (7.7) by

$$
\left.\frac{\partial B}{\partial t}+\nabla_{x} \times \nabla_{x} \times H+\nabla_{y} \times \nabla_{y} \times H=f \quad \text { in } \mathbb{R}^{3} \times \mathcal{Y} \times\right] 0, T[.
$$

In this case the energy integral would yield the square-integrability of $\nabla_{x} \times H$ and $\nabla_{y} \times H$, but the two-scale extension of the Gauss law (7.4) would be ruled out.

\section{References}

[1] G. Allaire, Homogenization and two-scale convergence, SIAM J. Math. Anal. 23 (1992), 1482-1518.

[2] G. Allaire, "Shape Optimization by the Homogenization Method", Springer, New York, 2002.

[3] T. Arbogast, J. Douglas, U. Hornung, Derivation of the double porosity model of single phase flow via homogenization theory, SIAM J. Math. Anal. 21 (1990), 823-836.

[4] J.-P. Aubin, Un théorème de compacité, C.R. Acad. Sci. Paris, Série I 256 (1963), 50425044.

[5] N. Bakhvalov and G. Panasenko, "Homogenisation: Averaging Processes in Periodic Media”, Kluwer, Dordrecht, 1989.

[6] G. Bensoussan, J..L. Lions and G. PApanicolaou, "Asymptotic Analysis for Periodic Structures", North-Holland, Amsterdam, 1978.

[7] A. Bourgeat, S. LuCKhaus and A. Mikelić, Convergence of the homogenization process for a double-porosity model of immiscible two-phase flow, SIAM J. Math. Anal. 27 (1996), 1520-1543.

[8] H. Brezis, "Opérateurs Maximaux Monotones et Semi-Groupes de Contractions dans les Espaces de Hilbert”, North-Holland, Amsterdam, 1973.

[9] M. BRIANE and J. CASADO-DÍAZ, Lack of compactness in two-scale convergence, SIAM J. Math. Anal. 37 (2005), 343-346.

[10] D. Cioranescu, A. Damlamian and G. Griso, Periodic unfolding and homogenization, C.R. Acad. Sci. Paris, Sér. I 335 (2002), 99-104.

[11] D. Cioranescu and P. Donato, "An Introduction to Homogenization", Oxford Univ. Press, New York, 1999.

[12] I. Ekeland and R. Temam, "Analyse Convexe et Problèmes Variationnelles", Dunod Gauthier-Villars, Paris, 1974.

[13] J.-B. Hiriart-UrRuty and C. Lemarechal, "Convex Analysis and Optimization Algorithms", Springer, Berlin, 1993.

[14] J. D. JACKSON, "Classical Electrodynamics”, Wiley, Chichester, 1962.

[15] V. V. JikOV, S. M. KozLOV and O.A. OlEINIK, "Homogenization of Differential Operators and Integral Functionals", Springer, Berlin, 1994.

[16] M. Lenczner, Homogénéisation d'un circuit électrique, C.R. Acad. Sci. Paris, Ser. II 324 (1997), 537-542.

[17] J. L. LiOns, "Quelques Méthodes de Résolution des Problèmes aux Limites non Linéaires”, Dunod, Paris, 1969.

[18] F. Murat, Compacité par compensation, Ann. Scuola Norm. Sup. Pisa Cl. Sci. (4) 5 (1978), 489-507. 
[19] F. Murat, Compacité par compensation. II, In: "Proceedings of the International Meeting on Recent Methods in Nonlinear Analysis" held in Rome in 1978, E. De Giorgi and E. Magenes (eds.), Pitagora, Bologna, 1979, 245-256.

[20] F. MURAT, A survey on compensated compactness, In: "Contributions to Modern Calculus of Variations”, Bologna, 1985, Pitman Res. Notes Math. Ser., Vol. 148, Longman, Harlow, 1987, 145-183.

[21] F. Murat and L. TARTAR, $H$-convergence, In: "Topics in the Mathematical Modelling of Composite Materials", A. Cherkaev and R. Kohn (eds.), Birkhäuser, Boston 1997, 21-44.

[22] G. NGUETSEng, A general convergence result for a functional related to the theory of homogenization, SIAM J. Math. Anal. 20 (1989), 608-623.

[23] G. NGUetseng, Asymptotic analysis for a stiff variational problem arising in mechanics, SIAM J. Math. Anal. 21 (1990), 1394-1414.

[24] E. SancheZ-Palencia, "Non-Homogeneous Media and Vibration Theory", Springer, New York, 1980.

[25] J. Simon, Compact sets in the space $L^{p}(0, T ; B)$, Ann. Mat. Pura Appl. 146 (1987), 6596.

[26] L. TARTAR, "Course Peccot", Collège de France, Paris 1977, unpublished, partially written in [21].

[27] L. TARTAR, Une nouvelle méthode de résolution d'équations aux dérivées partielles non linéaires, In: “Journées d'Analyse Non linéaire”, Springer, Berlin, 1978, 228-241.

[28] L. TARTAR, Compensated compactness and applications to partial differential equations, In: "Nonlinear Analysis and Mechanics: Heriott-Watt Symposium", Vol. IV, R. J. Knops (ed.), 1979, 136-212.

[29] L. TARTAR, Mathematical tools for studying oscillations and concentrations: from Young measures to H-measures and their variants, In: "Multiscale Problems in Science and Technology", N. Antonić, C. J. van Duijn, W. Jäger and A. Mikelić (eds.), Springer, Berlin, 2002, $1-84$.

[30] A. Visintin, Some properties of two-scale convergence, Atti Accad. Naz. Lincei Cl. Sci. Fis. Mat. Natur. Rend. Lincei (9) Mat. Appl. 15 (2004), 93-107.

[31] A. Visintin, Towards a two-scale calculus, ESAIM Control Optim. Calc. Var. 12 (2006), 371-397.

[32] A. Visintin, Homogenization of doubly-nonlinear equations, Atti Accad. Naz. Lincei Cl. Sci. Fis. Mat. Natur. Rend. Lincei (9) Mat. Appl. 17 (2006), 211-222.

[33] A. Visintin, Two-scale convergence of first-order operators, Z. Anal. Anwendungen 26 (2007), 133-164.

[34] A. Visintin, Two-scale convergence of some integral functionals, Calc. Var. Partial Differential Equations 29 (2007), 239-265.

Università degli Studi di Trento Dipartimento di Matematica via Sommarive, 14 38050 Povo TN (Italy) 